\title{
Review of Deep Learning Based Automatic Segmentation for Lung Cancer Radiotherapy
}

\author{
Xi Liu ${ }^{1}$, Kai-Wen Li $^{1,2}$, Ruijie Yang ${ }^{3 *}$ and Li-Sheng Geng ${ }^{1,2,4,5 *}$ \\ 1 School of Physics, Beihang University, Beijing, China, ${ }^{2}$ Beijing Advanced Innovation Center for Big Data-Based Precision \\ Medicine, School of Medicine and Engineering, Key Laboratory of Big Data-Based Precision Medicine, Ministry of Industry \\ and Information Technology, Beihang University, Beijing, China, ${ }^{3}$ Department of Radiation Oncology, Peking University Third \\ Hospital, Beijing, China, ${ }^{4}$ Beijing Key Laboratory of Advanced Nuclear Materials and Physics, Beihang University, Beijing, \\ China, ${ }^{5}$ School of Physics and Microelectronics, Zhengzhou University, Zhengzhou, China
}

OPEN ACCESS

Edited by:

An Liu,

City of Hope National Medical Center,

United States

Reviewed by:

Kuo Men,

Chinese Academy of Medical

Sciences and Peking Union

Medical College, China

Weiwei Zong,

Henry Ford Health System,

United States

*Correspondence:

Ruijie Yang

ruijyang@yahoo.com

Li-Sheng Geng

lisheng.geng@buaa.edu.cn

Specialty section:

This article was submitted to

Radiation Oncology,

a section of the journal

Frontiers in Oncology

Received: 30 May 2021

Accepted: 21 June 2021

Published: 08 July 2021

Citation:

Liu X, Li K-W, Yang R and Geng L-S (2021) Review of Deep Learning Based Automatic Segmentation for Lung Cancer Radiotherapy.

Front. Oncol. 11:717039. doi: 10.3389/fonc.2021.717039
Lung cancer is the leading cause of cancer-related mortality for males and females. Radiation therapy (RT) is one of the primary treatment modalities for lung cancer. While delivering the prescribed dose to tumor targets, it is essential to spare the tissues near the targets - the so-called organs-at-risk (OARs). An optimal RT planning benefits from the accurate segmentation of the gross tumor volume and surrounding OARs. Manual segmentation is a time-consuming and tedious task for radiation oncologists. Therefore, it is crucial to develop automatic image segmentation to relieve radiation oncologists of the tedious contouring work. Currently, the atlas-based automatic segmentation technique is commonly used in clinical routines. However, this technique depends heavily on the similarity between the atlas and the image segmented. With significant advances made in computer vision, deep learning as a part of artificial intelligence attracts increasing attention in medical image automatic segmentation. In this article, we reviewed deep learning based automatic segmentation techniques related to lung cancer and compared them with the atlas-based automatic segmentation technique. At present, the auto-segmentation of OARs with relatively large volume such as lung and heart etc. outperforms the organs with small volume such as esophagus. The average Dice similarity coefficient (DSC) of lung, heart and liver are over 0.9, and the best DSC of spinal cord reaches 0.9. However, the DSC of esophagus ranges between 0.71 and 0.87 with a ragged performance. In terms of the gross tumor volume, the average DSC is below 0.8. Although deep learning based automatic segmentation techniques indicate significant superiority in many aspects compared to manual segmentation, various issues still need to be solved. We discussed the potential issues in deep learning based automatic segmentation including low contrast, dataset size, consensus guidelines, and network design. Clinical limitations and future research directions of deep learning based automatic segmentation were discussed as well.

Keywords: lung cancer, deep learning, automatic segmentation, organs-at-risk, radiotherapy 


\section{INTRODUCTION}

Cancer is becoming the leading cause of death and the most prominent obstacle to life expectancy increases in all countries. According to GLOBOCAN 2020, it is estimated that 19.3 million new cancer cases and 9.96 million cancer deaths occurred in 2020. Lung cancer, accounting for $11.4 \%$ of all new cases, is the second most common cancer. Meanwhile, it ranks first among the cancer-related mortality worldwide, accounting for $18.0 \%$ of the total cancer death (1).

In order to control the malignant tumors and improve the quality of life of cancer patients, various cancer treatment methods have gradually emerged in addition to surgical resection $(2,3)$, such as chemotherapy (4), radiotherapy (5-7), thermotherapy (8-10), immunotherapy $(11,12)$ and so on. With radiation therapy (RT) witnessing tremendous advancements in recent years, RT plays a crucial role in lung cancer treatment $(6,7,13-15)$. The success of RT depends on accurate irradiating the tumor targets while sparing the organs-at-risk (OARs) and avoiding RT-related complications. Accordingly, it is vital to segment the gross tumor volume (GTV) and OARs accurately in the RT treatment planning to deliver the prescription dose to the GTV.

Manual segmentation of the GTV and OARs is a laborious and tedious process for radiation oncologists, which could result in significant delays of RT treatment and low survival rates, especially in clinics with inadequate resources. Furthermore, the quality of manual segmentation relies on the prior knowledge and experience of the radiation oncologists. Even if they segmented the GTV and OARs according to the same guidelines, inconsistencies may still exist in the segmentation for both inter- and intra-observers. On the other hand, the automatic segmentation technique has the potential to provide efficient and accurate results $(16,17)$. It can not only shorten the time needed to exploit the anatomy but also allow experts to devote time to optimize RT treatment planning so that the OARs could be less irradiated. In recent years, various image segmentation techniques have been proposed, resulting in more accurate and efficient image segmentation for clinical diagnosis and treatment (18-24).

Traditional automatic segmentation techniques usually segment the target depending on the shallow features of the image such as grayscale, texture, gradient, etc. In traditional automatic segmentation techniques, the common methods are Thresholding Method (25, 26), Atlas Method (27), and Region Growing Method (28) etc. Based on the target and background needed to be segmented, appropriate grayscale thresholding is selected. According to the selected thresholding, all pixels in the image to be segmented are classified into two categories, viz target and background, to perform the segmentation task. But when the grayscale difference between the image background and the target is not significant, it is difficult to segment the image accurately and efficiently (29). The Atlas Method registers the new input image to the reference image known as an atlas template, and then the labels in the atlas templates are propagated to the new input image to finalize the delineating task (30). However, the performance of the Atlas Method is heavily reliant upon the registration algorithms and the quality of the selected atlas templates (31). The Region Growing Method manually defines sub-regions in advance, then merges the adjacent pixels with similar attributes to the pre-defined region, and finally achieves the segmentation of the target region from the background (32). Nevertheless, the Region Growing Method lacks objectivity owing to the manual selection of sub-regions. Moreover, when the color feature or location information of the organs to be segmented is similar to that of other organs, the segmentation accuracy is usually not sufficiently high.

With the development of deep learning, deep learning-based models have shown superior capabilities in medical image autosegmentation (33). Deep learning models learn feature representation independently and utilize the learned highdimensional abstraction to finalize segmentation tasks without manual intervention (20). Recently, several studies have proposed various deep learning based automatic segmentation techniques for lung cancer (34-42). There is not yet a review of deep learning based automatic segmentation techniques for lung cancer radiotherapy. This manuscript aims to comprehensively review the deep learning based automatic segmentation techniques on lung cancer radiotherapy. The current challenges, practical issues, and future research directions of automatic segmentation are also discussed.

\section{DEEP LEARNING BASED AUTOMATIC SEGMENTATION}

\section{Basis of Deep Learning}

With significant advances in computing technique and data accumulation, deep learning as a branch of artificial intelligence is attracting increasing attention in image automatic segmentation $(29,33,43)$. Along with the continual increase of the model depth, deep learning can represent more complex phenomena by hierarchically extracting features of the input data via the hidden layers and by repeatedly training the network with the input data, such as convolutional neural networks (CNNs) (44), fully convolutional networks (FCNs) (45), and U-Net (46).

As shown in Figure 1, CNNs (44) are generally feedforward neural networks composed of convolutional layers, pooling layers, and fully connected layers. In principle, CNNs allow to classify each individual pixel in the image, whereas the training of CNNs becomes time-consuming and computationally expensive. Although the CNN models can automatically extract image features, the pooling layers also reduce the image's resolution while shrinking the size of the feature maps. Also, the fully connected layers have a fixed number of nodes, which limits the size of the input images.

In 2015, Long et al. (45) proposed fully convolutional networks (FCNs) based on the improvement of CNNs. FCNs replace the final fully-connected layers of $\mathrm{CNNs}$ with the convolutional layers so that FCNs can accept input images of any size. The skip connections in FCNs improve the efficiency of image segmentation and combine the context information of the image simultaneously. Figure $\mathbf{2}$ is a typical FCN. However, the 


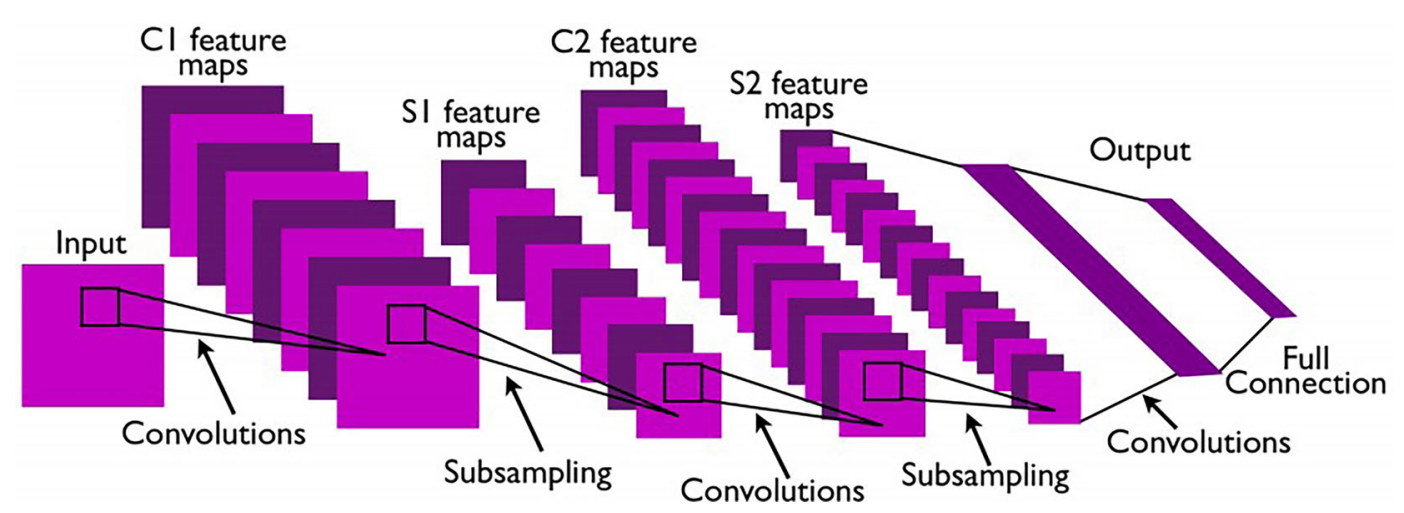

FIGURE 1 | Architecture of a classic CNN (44).

problem is that the multiplier used in the FCNs upsampling operation is too large, resulting in the loss of segmentation accuracy and insufficient integration of context information.

To solve this problem, Ronneberger et al. (46) proposed the U-Net architecture (as illustrated in Figure 3), which uses the same number of convolutional layers in upsampling and downsampling. In addition, a skip connection exists between each level of the upsampling layer and the correspondingly downsampling layer, which enables the features extracted by the downsampling layer to be passed to the upsampling layer. The above-mentioned two improvements make U-Net more accurate in the aspect of pixel positioning and segmentation.

\section{Segmentation of OARs and GTV for Lung Cancer}

The pathological characteristics of lung cancer are more complex compared with other malignant tumors. Early clinical diagnosis of lung cancer is often difficult, so that the majority of patients diagnosed with lung cancer have reached the advanced stage. The five-year survival rate of patients with advanced stage lung cancer is less than $15 \%$, but this value can reach $40 \sim 70 \%$ if diagnosed at the early stage (47). Therefore, the early diagnosis and treatment of lung cancer is the key to improving the curative ratio. Employing deep learning in clinical practice may potentially shorten the unnecessary time and alleviate the workload of relevant staff (48). In recent years, several deep learning based automatic segmentation techniques have been proposed successively $(35,49-56)$. In this section, studies related to the deep learning based automatic segmentation of the OARs and GTV in lung cancer are discussed and compared. A manual searching with keywords "lung cancer, automatic segmentation, and deep learning" was carried out on three academic electronic databases viz. Web of Science, PubMed, and IEEE Xplore. Studies published from 2018 2020 are selected in this review.

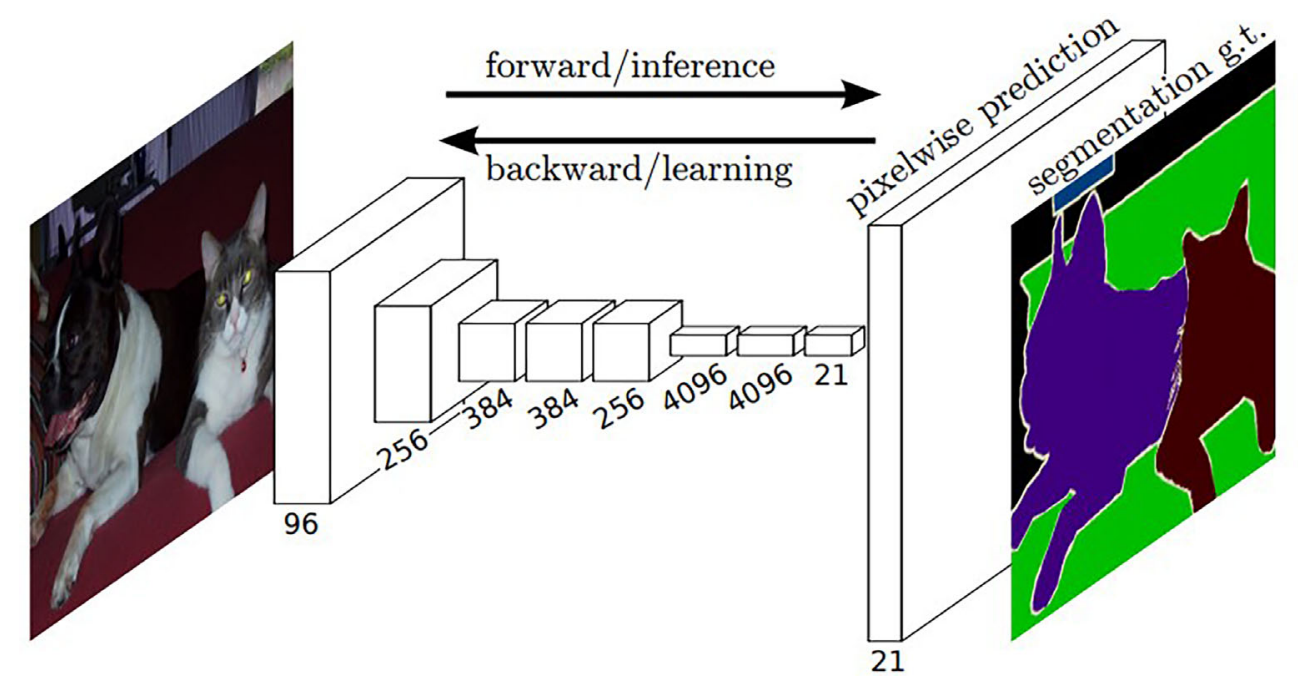

FIGURE 2 | Architecture of a typical FCN (45). The white boxes represent multi-channel feature maps after the convolutional operation. 


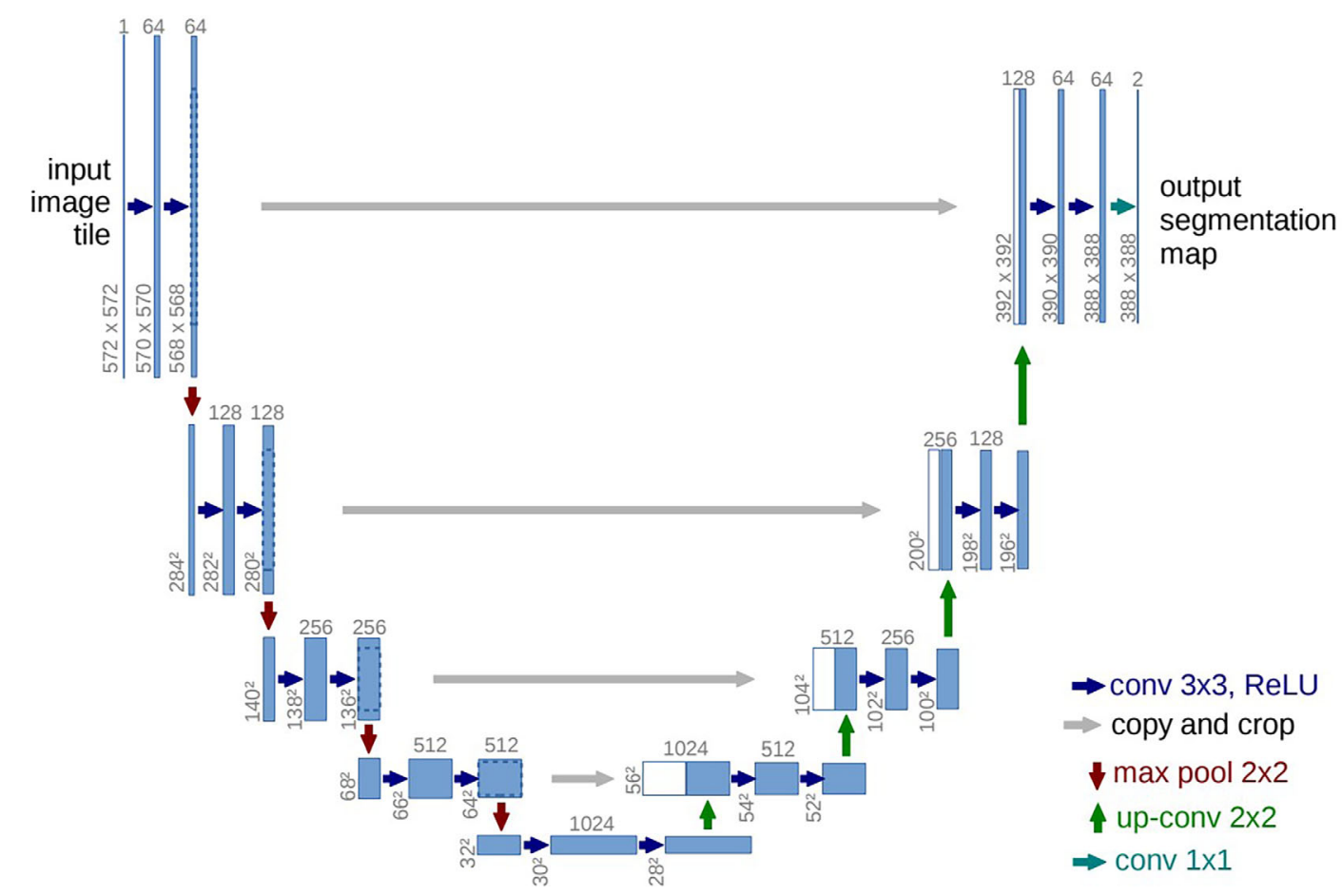

FIGURE 3 | Architecture of a conventional U-Net (46). The blue boxes represent multi-channel feature maps, and the white boxes correspond to the copy of feature maps in the encoder branch. The arrows of different colors represent various operations. The number provided on the top of the box represents the number of channels, and the $x-y$-size of feature map is denoted at the lower left edge of the box.

\section{OARs Segmentation}

The precise segmentation of OARs is of vital importance to optimize the delivery of decreased dose to normal tissues, and it strongly affects the quality and outcome in lung cancer RT. Some studies have been published with regard to the segmentation of OARs in lung cancer utilizing deep learning algorithms.

In 2018, Zhao et al. (35) proposed a FCN-based network to segment lung with various diseases. In their design, they introduced a multi-instance loss to facilitate updating the most input-related convolution kernels during iterative training, and employed a conditional adversary loss to assist in correcting the lung segmentation mask. The DSC they achieved over three datasets was $0.9176,0.9613$, and 0.9793 , respectively. Agnes et al. (57) trained a CNN-based network to segment lung in low-dose chest computed tomography (CT) images. They reported a mean DSC of 0.95 on LIDC-IDRI database (58). Zhu et al. (59) developed a CNN-based deep learning algorithm to automatically segment multiple thoracic OARs, including lungs, heart, spinal cord, esophagus, and liver. In their design, the network architecture was adapted from U-Net, but replacing the convolutional layer with a residual convolutional unit. In their method, the DSC of lungs, heart, spinal cord, esophagus, and liver was $0.95 \pm 0.01,0.91 \pm 0.03,0.79 \pm 0.03,0.71 \pm 0.05$, and $0.89 \pm 0.02$, respectively.

In 2019, Dong et al. (38) proposed a U-Net-GAN strategy to automatically contour left and right lungs, spinal cord, esophagus and heart. Their design adopted the architecture of generative adversarial network (GAN), employing the U-Nets as generators and the FCNs as discriminators. They achieved a DSC of $0.97 \pm 0.01,0.97 \pm 0.01,0.90 \pm 0.04,0.87 \pm 0.05$, and $0.75 \pm$ 0.08 for the left lung, right lung, spinal cord, heart, and esophagus, respectively. Correspondingly, the mean surface distance $(\mathrm{MSD})$ was $0.61 \pm 0.73,0.65 \pm 0.53,0.38 \pm 0.27$, $1.49 \pm 0.85$, and $1.05 \pm 0.66 \mathrm{~mm}$. The average sensitivity of the proposed method was $0.74 \sim 0.97$, with the best for the lung and the worst for the esophagus. Additionally, they compared the performance of U-Net with and without the adversarial network. They concluded that with the assistance of the adversarial network, the segmentation accuracy was improved, and the biggest improvement was found for the spinal cord.

Later, Feng et al. (39) developed another novel segmentation model based on 3D U-Net for the automatic segmentation of five thoracic OARs, including left and right lungs, heart, esophagus and spinal cord. In their model, given that each organ has a relatively fixed position within the CT images, they firstly cropped the original $3 \mathrm{D}$ images into smaller patches ensuring each patch containing only one organ to be segmented. Secondly, for each organ, an individual $3 \mathrm{D}$ U-Net was trained to segment the organ from the cropped patches. The individual segmentation results were resampled and integrated together to generate the final segmentation results. According to their testing, the model segmented the OARs with a mean DSC of $0.893,0.972,0.979,0.925,0.726$ for the spinal cord, right lung, left lung, heart and esophagus, respectively. The MSDs were as follows: (spinal cord: $0.662 \pm 0.248 \mathrm{~mm}$, right lung: $0.933 \pm$ 
$0.574 \mathrm{~mm}$, left lung: $0.586 \pm 0.285 \mathrm{~mm}$, heart: $2.297 \pm 0.492 \mathrm{~mm}$, esophagus: $2.341 \pm 2.380 \mathrm{~mm}$ ).

In the same year, Trullo et al. (60) organized a competition called SegTHOR on the theme "Automatic segmentation of Organs-at-risk in Thoracic CT images". In the competition, various segmentation techniques based on different frameworks were proposed to automatically delineate four OARs: heart, aorta, trachea, esophagus. Among all the techniques based on CNN architecture, Harten et al. (61) obtained the best performance in terms of DSC (esophagus: 0.84, heart: 0.94, trachea: 0.91, and aorta: 0.93) and Hausdorff distance (HD) (esophagus: $3.4 \mathrm{~mm}$, heart: $2.0 \mathrm{~mm}$, trachea: $2.1 \mathrm{~mm}$, and aorta: $2.7 \mathrm{~mm}$ ). They combined a $2 \mathrm{D} \mathrm{CNN}$ with a $3 \mathrm{D}$ CNN to segment the OARs. The $2 \mathrm{D} \mathrm{CNN}$ containing dilated convolutions performed multi-class segmentation while the 3D CNN containing residual blocks performed multi-label segmentation, promoting additional diversity in the networks.

Among all the techniques based on U-Net architecture, $\mathrm{He}$ et al. (62) obtained the highest value of DSC and lowest HD for esophagus (DSC: 0.8594; HD: 0.2743), heart (DSC: 0.9500; HD: 0.1383 ) and aorta (DSC: 0.9484 ; HD: 0.1129 ). They proposed a uniform U-like encoder-decoder architecture abstracted from the U-Net and trained it under the multi-task learning schema. Commonly used network architecture such as ResNet and DenseNet could be involved in the encoder part by eliminating their linearly connected layers, and the encoder could adopt the transfer learning under this design. It is the transfer learning that shortens the training time and boots the performance of the network. With regard to the trachea, Vesal et al. (63) achieved a better performance than He et al. with a DSC of 0.926 and an HD of $0.193 \mathrm{~mm}$. They modified the 2D U-Net mainly in two aspects. Firstly, dilated convolutions were introduced to expand the receptive fields so that both local and global information was used efficiently without increasing the network complexity. Additionally, to better incorporate multi-scale image features, the convolution layers were replaced with the residual convolution layers in the encoder branch.

Among all the techniques based on V-Net architecture, the best result for the esophagus (DSC: 0.8651; HD: $0.2590 \mathrm{~mm}$ ), heart (DSC: 0.9536 ; HD: $0.1272 \mathrm{~mm}$ ), trachea (DSC: 0.9276; HD: $0.1453 \mathrm{~mm}$ ) and aorta (DSC: 0.9464; HD: $0.1209 \mathrm{~mm}$ ) was obtained by Han et al. (40) who was the winner of the SegTHOR competition. Based on V-Net, they proposed a novel framework called multi-resolution VB-Net. The network consists of two parts: (a) the contraction path on the left side to extract high-level contextual information of the input data employing convolution and downsampling; (b) the expansion path on the right side to integrate high-level contextual information with detailed local information via skip connections to improve the accuracy of the outputs. They utilized the bottleneck structure to replace the conventional convolutional layers inside the upsampling and downsampling processes. Furthermore, to reduce the GPU memory and computation cost, they adopted a multi-resolution strategy. They trained two VB-Nets separately, one in the coarse resolution to roughly get the location of the ROI for each organ and the other in the fine resolution to accurately delineate the OAR boundaries from the detected ROI.
In 2020, Zhang et al. (64) established a CNN network based on ResNet-101 for automatically segmenting the OARs, including lungs, esophagus, heart, liver, and spinal cord. They reported a mean DSC of $0.948,0.943,0.821,0.893,0.937$ and 0.732 for the left lung, right lung, spinal cord, heart, liver, and esophagus, respectively. Correspondingly, the MSD was $1.10 \pm 0.15,2.23 \pm$ $2.33,0.87 \pm 0.21,1.65 \pm 0.48,2.03 \pm 1.49$ and $1.38 \pm 0.44 \mathrm{~mm}$.

$\mathrm{Hu}$ et al. (65) used Mask R-CNN architecture combined with supervised (Bayes, Support Vectors Machine) and unsupervised (K-means and Gaussian Mixture Models) machine learning methods to segment lungs on CT images automatically. Mask R-CNN consists of two stages: (a) a Region Proposal Network to generate candidate object bounding boxes and predict the classes of objects; (b) while predicting the class and box offset, an FCN to generate a binary mask for each detected object. They concluded that the method combining Mask R-CNN with the K-means kernel generated the best results for lung segmentation with a DSC of $97.33 \pm 3.24 \%$ and a sensitivity of $96.58 \pm 8.58 \%$.

Apart from various $\mathrm{CNN}$ models, GAN models have also been utilized for image segmentation. Tan et al. (66) proposed a new schema called LGAN for lung segmentation based on the architecture of GAN. Meanwhile, a novel loss function based on the Earth Mover distance was used in their schema. For this schema, a generative network (generator) is constructed to produce the lung mask, and a discriminative network (discriminator) is constructed to differentiate the generated synthetic maps from the ground truth. The generator and discriminator are trained sequentially and iteratively in a competing way to boost the performance of the other, which assists the generator to generate lung segmentation results that cannot be differentiated from the ground truth. After exploring various discriminator designs for lung segmentation, they achieved the best performance by designing the discriminative network as a regression network. This proposal had an Intersection over Union (IOU) of 0.923 and an HD of $3.380 \mathrm{~mm}$ on the LIDC-IDRI dataset in which the patients were selected from the public database founded by the Lung Image Database Consortium and Image Database Resource Initiative. They also evaluated the proposal on another private dataset, achieving an IOU of 0.938 and an HD of $2.812 \mathrm{~mm}$. Considering both using the LIDC-IDRI dataset, a comparison between LGAN and U-Net (46) had been conducted. According to the results of the paper, the DSC of LGAN and U-Net in [mean, median] form were [0.970 \pm 0.59 , $0.9845]$ vs. [0.985 $\pm 0.03,0.9864]$. A better DSC indicates that LGAN outperforms commonly used U-Net.

Pawar et al. (67) developed a deep learning algorithm to effectively segment lung, which is denoted as LungSeg-Net. LungSeg-Net is similar to GAN, including two networks, viz. generator and discriminator. The generator is composed of three major components: (a) encoder block to extract feature maps; (b) multi-scale dense feature extraction module to extract multi-scale features from the set of encoded feature maps; (c) decoder block to generate the output lung segmentation map from the multi-scale features. They compared the performance of the proposed LungSegNet with the existing state-of-the-art CNNs viz. U-Net (46), ResNet (68), VGG16 (69). The DSC of lung achieved with the proposed network ranges between 0.9140 and 0.9899 on average. 
They concluded that the LungSeg-Net showed considerable performance improvement compared to other CNNs in the lung segmentation with different interstitial lung disease patterns.

At present, OARs segmentation is currently limited to delineate the whole organ in the majority of studies related to automatic segmentation. Yet, there is evidence suggesting that dose to sensitive cardiac substructures may give rise to cardiac toxicities (70-72) involving cardiomyopathy, coronary artery disease etc. (73). Especially, coronary artery calcification onset has been more relevant to the maximum dose to the left anterior descending artery, compared to the mean heart dose (74). However, because of the limited ability to contour these sensitive cardiac substructures, these dose thresholds are unavailable. Recently, Morris et al. (75) explored the accurate segmentation of twelve cardiac substructures, including chambers, great vessels, coronary arteries, etc. They proposed a 3D U-Net combined with a fully connected conditional random field to automatically segment cardiac substructures. Eventually, they obtained acceptable segmentations for chambers (DSC: $0.88 \pm 0.03$ ), great vessels (DSC: $0.85 \pm 0.03$ ), and pulmonary veins (DSC: $0.77 \pm 0.04$ ), compared to inferior performance on coronary arteries (DSC: $0.50 \pm 0.14$ ). In terms of further refinement of coronary artery segmentation, the author stated that utilizing conditional random fields as RNNs may be worth of studying.
It's also worth noting that most works focused on segmenting OARs using deep learning based algorithms in single energy CT images. On the other hand, dual energy CT which enables to acquire two different CT images concurrently (76) could supply higher contrast and more information about differences between tissues, compared with single energy CT. Therefore, using dual energy CT as input of deep learning network may help achieve more accurate segmentation $(77,78)$. Chen et al. (78) designed four 3D FCNs on basis of U-Net and ResNet for automatically segmenting the OARs using dual energy CT images. The four networks merged the extra information into the network in different ways: (a) linearly combining dual energy images into one mixed image as the input; (b) using the dual energy images as two channels of the same input; (c) extracting features of the low energy image and the high energy image separately and fusing them at the bottom of the U-Net; (d) handling the low energy image and the high energy image separately and fusing the prediction results into one finally. According to their test results, the best mean DSC were $97.5 \pm 0.64 \%, 97.6 \pm 1.61 \%$, and $96.2 \pm 1.64 \%$, for the left lung, right lung, and liver, separately.

Table 1 is a brief summary of the aforementioned works. Tables 2-6 shows the comparison of selected works on the segmentation of different OARs.

TABLE 1 | Selected works on deep learning-based automated segmentation of OARs for lung cancer.

\begin{tabular}{|c|c|c|c|c|}
\hline Reference & Year & Networks & OARs & Research Highlight \\
\hline Zhao et al. (35) & 2018 & FCN & lung & $\begin{array}{l}\text { introducing a multi-instance loss and a conditional adversary loss to } \\
\text { facilitate more correct segmentation }\end{array}$ \\
\hline Agnes et al. (57) & 2018 & U-Net & lung & $\begin{array}{l}\text { exploring the performance of different convolutional network configurations, and } \\
\text { comparing proposed model with other methods such as the thresholding method etc. }\end{array}$ \\
\hline Zhu et al. (59) & 2018 & U-Net & $\begin{array}{l}\text { lung, heart, esophagus, spinal cord, } \\
\text { and liver }\end{array}$ & replacing the common convolutional layers with residual convolution units \\
\hline Dong et al. (38) & 2019 & U-net-GAN & $\begin{array}{l}\text { left lung, right lung, heart, } \\
\text { esophagus, and spinal cord }\end{array}$ & $\begin{array}{l}\text { proposing a } 2.5 \mathrm{D} \text { patch-based GAN to delineate the left lung, right lung, and } \\
\text { heart, two 3D patch-based GAN to delineate esophagus and spinal cord separately }\end{array}$ \\
\hline Feng et al. (39) & 2019 & U-Net & $\begin{array}{l}\text { left lung, right lung, heart, } \\
\text { esophagus, and spinal cord }\end{array}$ & $\begin{array}{l}\text { developing two 3D U-Nets, one for localizing each OAR and the other for individually } \\
\text { segmenting each OAR }\end{array}$ \\
\hline Harten et al. (61) & 2019 & CNN & heart, aorta, trachea, esophagus & $\begin{array}{l}\text { combining a 2D CNN which utilizes dilated convolutions with a 3D CNN } \\
\text { which employs residual blocks }\end{array}$ \\
\hline He et al. (62) & 2019 & adapted U-Net & heart, aorta, trachea, esophagus & $\begin{array}{l}\text { proposing a U-like architecture in which the encoder could set diverse network } \\
\text { framework, and training it under the multi-task learning schema }\end{array}$ \\
\hline Vesal et al. (63) & 2019 & U-Net & heart, aorta, trachea, esophagus & $\begin{array}{l}\text { employing dilated convolutions in the bottleneck of a } 2 \mathrm{D} U \text {-Net styled network and } \\
\text { residual connections in the encoder branch }\end{array}$ \\
\hline Han et al. (40) & 2019 & V-Net & heart, aorta, trachea, esophagus & $\begin{array}{l}\text { proposing a multi-resolution VB-Net architecture by replacing the convolutional } \\
\text { layers inside the V-Net with a bottleneck framework }\end{array}$ \\
\hline Zhang et al. (64) & 2020 & CNN & $\begin{array}{l}\text { left lung, right lung, heart, } \\
\text { esophagus spinal cord, and liver }\end{array}$ & $\begin{array}{l}\text { establishing a dilated CNN structure based on ResNet-101, and comparing its } \\
\text { performance with atlas-based and manual segmentation }\end{array}$ \\
\hline Hu et al. (65) & 2020 & Mask R-CNN & lung & $\begin{array}{l}\text { using a Mask R-CNN combined with supervised and unsupervised machine } \\
\text { learning methods to segment the lung }\end{array}$ \\
\hline Tan et al. (66) & 2020 & GAN & lung & $\begin{array}{l}\text { proposing a GAN-based architecture combing a novel loss function based on } \\
\text { the Earth Mover distance for lung segmentation }\end{array}$ \\
\hline Pawar et al. (67) & 2020 & C-GAN & lung & $\begin{array}{l}\text { introducing a c-GAN structure that used multi-scale dense feature extraction } \\
\text { blocks for the lung segmentation with different interstitial lung disease patterns }\end{array}$ \\
\hline Morris et al. (75) & 2020 & U-Net & cardiac substructures & $\begin{array}{l}\text { proposing a 3D U-Net combined with fully connected conditional random fields to } \\
\text { segment twelve cardiac substructures }\end{array}$ \\
\hline Chen et al. (78) & 2020 & FCN & $\begin{array}{l}\text { left lung, right lung, liver, spleen, left } \\
\text { kidney, and right kidneys }\end{array}$ & $\begin{array}{l}\text { proposing a 3D FCN based on the U-Net and ResNet to segment OARs } \\
\text { in dual energy CT images }\end{array}$ \\
\hline
\end{tabular}

FCN, fully convolutional network; GAN, generative adversarial network; OAR, organ-at-risk; 3D, three dimensional; 2D, two dimensional; CNN, convolutional neural network; R-CNN, Region- convolutional neural network; c-GAN, conditional generative adversarial network. 
TABLE 2 | Comparison of selected works on segmentation of lung.

\begin{tabular}{|c|c|c|c|c|c|c|c|c|c|}
\hline \multirow[t]{2}{*}{ Reference } & \multirow[t]{2}{*}{ Year } & \multirow[t]{2}{*}{ Networks } & \multicolumn{7}{|c|}{ Evaluation Metrics } \\
\hline & & & DSC & IOU & HD (mm) & 95\%HD (mm) & MSD (mm) & Sensitivity & Specificity \\
\hline Zhao et al. (35) & 2018 & FCN & $\begin{array}{l}\text { LIDC: } 0.92 \\
\text { CLEF: } 0.96 \\
\text { HUG: } 0.98\end{array}$ & - & - & - & - & - & - \\
\hline Agnes et al. (57) & 2018 & U-Net & $0.95 \pm 0.03$ & - & - & - & - & $0.95 \pm 0.03$ & $0.99 \pm 0.01$ \\
\hline Zhu et al. (59) & 2018 & adapted U-Net & $0.95 \pm 0.01$ & - & - & $7.96 \pm 2.57$ & $1.93 \pm 0.51$ & - & - \\
\hline Dong et al. (38) & 2019 & U-net-GAN & $\begin{array}{r}\text { Left: } 0.97 \pm 0.01 \\
\text { Right: } 0.97 \pm 0.01\end{array}$ & - & - & $\begin{array}{l}2.07 \pm 1.93 \\
2.50 \pm 3.34\end{array}$ & $\begin{array}{l}0.61 \pm 0.73 \\
0.65 \pm 0.53\end{array}$ & $\begin{array}{l}0.97 \pm 0.02 \\
0.96 \pm 0.02\end{array}$ & $\begin{array}{l}0.9989 \pm 0.0010 \\
0.9992 \pm 0.0007\end{array}$ \\
\hline Feng et al. (39) & 2019 & 3D U-Net & $\begin{array}{r}\text { Left: } 0.98 \pm 0.01 \\
\text { Right: } 0.97 \pm 0.02\end{array}$ & - & - & $\begin{array}{l}2.10 \pm 0.94 \\
3.96 \pm 2.85\end{array}$ & $\begin{array}{l}0.59 \pm 0.29 \\
0.93 \pm 0.57\end{array}$ & - & - \\
\hline Zhang et al. (64) & 2020 & ResNet-101 & $\begin{array}{r}\text { Left: } 0.95 \pm 0.01 \\
\text { Right: } 0.94 \pm 0.02\end{array}$ & - & - & - & $\begin{array}{l}1.10 \pm 0.15 \\
2.23 \pm 2.33\end{array}$ & - & - \\
\hline Hu et al. (65) & 2020 & Mask R-CNN & $0.97 \pm 0.03$ & - & - & - & - & $0.97 \pm 0.09$ & $0.9711 \pm 0.0365$ \\
\hline Tan et al. (66) & 2020 & GAN & - & 0.938 & 2.812 & - & - & - & - \\
\hline Chen et al. (78) & 2020 & 3D FCN & $\begin{array}{r}\text { Left: } 0.98 \pm 0.01 \\
\text { Right: } 0.98 \pm 0.02\end{array}$ & - & - & - & - & - & - \\
\hline
\end{tabular}

FCN, fully convolutional network; LIDC, Lung Image Database Consortium; CLEF, Conference and Labs of the Evaluation Forum; HUG, University Hospitals of Geneva; DSC, Dice similarity coefficient; IOU, intersection over Union; HD, Hausdorff distance; 95\%HD, 95\% Hausdorff distance; MSD, mean surface distance; R-CNN, Region- convolutional neural network; GAN, generative adversarial network.

\section{Lung Tumor Segmentation}

The usage of deep learning techniques assists radiation oncologists in segmenting lung on CT or magnetic resonance imaging (MRI) images with greater accuracy, consistency, and efficiency. Diverse network architectures are established by different authors in their published papers. Owing to the stateof-the-art performance of $\mathrm{CNNs}$ in challenging problems, for instance, computer vision, object detection, and image recognition, researchers gradually shift to using CNNs for the GTV segmentation and auxiliary diagnosis.

Inspired by CNN architectures, Wang et al. (36) introduced a new patient-specific adaptive convolutional neural network (A-net) for automatically contouring lung tumors seen on weekly MRI images. A-net mainly consists of three convolution blocks, three fully connected blocks, and one SoftMax layer. A dropout layer comes along with a fully connected layer in the last three levels, which was used to solve the potential over-fitting problem. $2 \mathrm{D}$ patches with a size of $3 \mathrm{~cm} \times 3 \mathrm{~cm}$ were cropped as inputs to Anet within the region of interest of the weekly MRI scans. A-net utilized the previous weekly MRI images and the segmentation of the GTV to train and update the network, and the current weekly MRI images were allocated as testing data. With this method, they obtained the segmentation results of the weekly MRI with a DSC and a precision of $0.82 \pm 0.10$ and $0.81 \pm 0.10$, respectively.

Zhang et al. (79) introduced another modified ResNet to segment the GTV of non-small cell lung cancer patients on the CT images. In this method, the deep features of the input data were effectively extracted using two different residual convolutional blocks. The feature maps generated at all levels of the ResNet were merged into a single output. This modification made shallow surface features fuse with the deep semantic features to generate dense pixel outputs. Utilizing the proposed modified ResNet, the average DSC level achieved is 0.73. A comparison between modified ResNet and U-Net had been conducted on the same dataset. They concluded that modified ResNet outperforms U-Net, because U-Net has a lower DSC with a mean value of 0.64 .

Given that the residual connections solely employed in ResNet do not eliminate the issue of poor localization and blurring arising from consecutive pooling operations, Pohlen et al. (80) proposed the full resolution residual neural network (FRRN) which passes features at full image resolution to each layer. In 2018, Jiang et al. (41) modified the FRRN and proposed two multiple resolution residually connected network (MRRN) architectures called incremental-MRRN and dense-MRRN to automatically segment lung tumors. When combining feature maps at multiple image resolutions and feature levels, a dense feature representation is simultaneously generated so that the performance of the MRNN on recovering the input image spatial resolution is better than other networks. The main difference between incremental-MRRN and dense-MRRN is that incremental-MRRN sequentially integrates higher spatial resolution information starting from the immediately previous residual stream, whereas dense-MRRN only residually integrates information from the immediate higher spatial resolution feature maps. In the work, the performance of different networks, including U-Net (46), SegNet (81) and FRRN (80) was compared with two MRNNs. The DSC, 95\%HD, sensitivity and precision of U-Net, SegNet, FRRN, incremental-MRRN and denseMRRN in three different datasets are shown in Table 7. According to their research results, it could be concluded that incrementalMRRN shows more robust performance than U-Net, SegNet, and FRRN.

In addition to single-modality segmentation, multi-modality cosegmentation have also been proposed $(82,83)$. Zhao et al. (82) proposed a novel scheme that utilizes both positron emission tomography (PET) and CT image information concurrently for lung tumor delineating. In their scheme, the network framework consisted of two parts namely multi-task training module and feature fusion module. The multi-task training module included two parallel sub-segmentation branches used for extracting features 
TABLE 3 | Comparison of selected works on segmentation of esophagus.

\begin{tabular}{|c|c|c|c|c|c|c|}
\hline \multirow[t]{2}{*}{ Reference } & \multirow[t]{2}{*}{ Year } & \multirow[t]{2}{*}{ Networks } & \multicolumn{4}{|c|}{ Evaluation Metrics } \\
\hline & & & DSC & HD (mm) & 95\%HD (mm) & MSD (mm) \\
\hline Zhu et al. (59) & 2018 & adapted U-Net & $0.71 \pm 0.05$ & - & $7.83 \pm 2.85$ & $2.18 \pm 0.80$ \\
\hline Dong et al. (38) & 2019 & U-net-GAN & $0.75 \pm 0.08$ & - & $4.52 \pm 3.81$ & $1.05 \pm 0.66$ \\
\hline Feng et al. (39) & 2019 & 3D U-Net & $0.73 \pm 0.09$ & - & $8.71 \pm 10.59$ & $2.34 \pm 2.38$ \\
\hline Harten et al. (61) & 2019 & CNN & $0.85 \pm 0.05$ & $3.4 \pm 2.3$ & - & - \\
\hline He et al. (62) & 2019 & U-Net with multi-task learning & 0.86 & 0.274 & - & - \\
\hline Vesal et al. (63) & 2019 & $2 \mathrm{D} U-\mathrm{Net}$ & 0.86 & 0.331 & - & - \\
\hline Han et al. (40) & 2019 & VB-Net & 0.87 & 0.259 & - & - \\
\hline Zhang et al. (64) & 2020 & ResNet-101 & $0.73 \pm 0.07$ & - & - & $1.38 \pm 0.44$ \\
\hline
\end{tabular}

DSC, Dice similarity coefficient; HD, Hausdorff distance; 95\%HD, 95\% Hausdorff distance; MSD, mean surface distance; GAN, generative adversarial network.

TABLE 4 | Comparison of selected works on segmentation of spinal cord.

\begin{tabular}{|c|c|c|c|c|c|}
\hline \multirow[t]{2}{*}{ Reference } & \multirow[t]{2}{*}{ Year } & \multirow[t]{2}{*}{ Networks } & \multicolumn{3}{|c|}{ Evaluation Metrics } \\
\hline & & & DSC & 95\%HD (mm) & $\operatorname{MSD}(\mathrm{mm})$ \\
\hline Zhu et al. (59) & 2018 & adapted U-Net & $0.79 \pm 0.03$ & $4.01 \pm 2.05$ & $1.25 \pm 0.23$ \\
\hline Dong et al. (38) & 2019 & U-net-GAN & $0.90 \pm 0.04$ & $1.19 \pm 0.46$ & $0.38 \pm 0.27$ \\
\hline Feng et al. (39) & 2019 & 3D U-Net & $0.89 \pm 0.04$ & $1.89 \pm 0.63$ & $0.66 \pm 0.25$ \\
\hline Zhang et al. (64) & 2020 & ResNet-101 & $0.82 \pm 0.05$ & - & $0.87 \pm 0.21$ \\
\hline
\end{tabular}

DSC, Dice similarity coefficient; HD, Hausdorff distance; 95\%HD, 95\% Hausdorff distance; MSD, mean surface distance; GAN, generative adversarial network.

TABLE 5 | Comparison of selected works on segmentation of heart.

\begin{tabular}{|c|c|c|c|c|c|c|}
\hline \multirow[t]{2}{*}{ Reference } & \multirow[t]{2}{*}{ Year } & \multirow[t]{2}{*}{ Networks } & \multicolumn{4}{|c|}{ Evaluation Metrics } \\
\hline & & & DSC & HD (mm) & 95\%HD (mm) & MSD (mm) \\
\hline Zhu et al. (59) & 2018 & adapted U-Net & $0.91 \pm 0.03$ & - & $7.98 \pm 4.56$ & $2.92 \pm 1.51$ \\
\hline Dong et al. (38) & 2019 & U-net-GAN & $0.87 \pm 0.05$ & - & $4.58 \pm 3.67$ & $1.49 \pm 0.85$ \\
\hline Feng et al. (39) & 2019 & 3D U-Net & $0.93 \pm 0.02$ & - & $6.57 \pm 1.50$ & $2.30 \pm 0.49$ \\
\hline Harten et al. (61) & 2019 & CNN & $0.95 \pm 0.01$ & $2.0 \pm 1.1$ & - & - \\
\hline He et al. (62) & 2019 & U-Net with multi-task learning & 0.95 & 0.138 & - & - \\
\hline Vesal et al. (63) & 2019 & $2 \mathrm{D} U-\mathrm{Net}$ & 0.94 & 0.226 & - & - \\
\hline Han et al. (40) & 2019 & VB-Net & 0.95 & 0.127 & - & - \\
\hline Zhang et al. (64) & 2020 & ResNet-101 & $0.89 \pm 0.05$ & - & - & $1.65 \pm 0.48$ \\
\hline
\end{tabular}

DSC, Dice similarity coefficient; HD, Hausdorff distance; 95\%HD, 95\% Hausdorff distance; MSD, mean surface distance; GAN, generative adversarial network.

TABLE 6 | Selected works on segmentation of other OARs (liver, aorta, trachea).

\begin{tabular}{|c|c|c|c|c|c|}
\hline \multirow[t]{2}{*}{ Reference } & \multirow[t]{2}{*}{ Year } & \multirow[t]{2}{*}{ Networks } & \multicolumn{3}{|c|}{ Evaluation Metrics } \\
\hline & & & DSC & HD (mm) & MSD (mm) \\
\hline Zhu et al. (59) & 2018 & adapted U-Net & Liver: $0.89 \pm 0.02$ & - & $3.21 \pm 0.93$ \\
\hline Zhang et al. (64) & 2020 & ResNet-101 & Liver: $0.94 \pm 0.03$ & - & $2.03 \pm 1.49$ \\
\hline Chen et al. (78) & 2020 & 3D FCN & Liver: $0.96 \pm 0.16$ & - & - \\
\hline \multirow[t]{2}{*}{ Harten et al. (61) } & 2019 & CNN & Aorta: $0.93 \pm 0.01$ & $2.7 \pm 3.6$ & - \\
\hline & & & Trachea: $0.91 \pm 0.02$ & $2.1 \pm 1.0$ & - \\
\hline \multirow[t]{2}{*}{ He et al. (62) } & 2019 & U-Net with multi-task learning & Aorta: 0.95 & 0.113 & - \\
\hline & & & Trachea: 0.92 & 0.182 & - \\
\hline \multirow[t]{2}{*}{ Vesal et al. (63) } & 2019 & 2D U-Net & Aorta: 0.94 & 0.297 & - \\
\hline & & & Trachea: 0.93 & 0.193 & - \\
\hline \multirow[t]{2}{*}{ Han et al. (40) } & 2019 & VB-Net & Aorta: 0.95 & 0.121 & - \\
\hline & & & Trachea: 0.93 & 0.145 & - \\
\hline
\end{tabular}

DSC, Dice similarity coefficient; HD, Hausdorf distance; 95\%HD, 95\% Hausdorff distance; MSD, mean surface distance; GAN, generative adversarial network. 
TABLE 7 | Comparison of different networks on segmentation of lung tumors in (41).

\begin{tabular}{|c|c|c|c|c|c|c|c|c|c|c|c|c|}
\hline \multirow[t]{2}{*}{ Networks } & \multicolumn{4}{|c|}{ TCIA dataset } & \multicolumn{4}{|c|}{ MSKCC dataset } & \multicolumn{4}{|c|}{ LIDC dataset } \\
\hline & DSC & $95 \% \mathrm{HD}$ & Sensitivity & Precision & DSC & $95 \% \mathrm{HD}$ & Sensitivity & Precision & DSC & $95 \% \mathrm{HD}$ & Sensitivity & Precision \\
\hline U-Net & 0.68 & 15.51 & 0.73 & 0.71 & 0.65 & 7.87 & 0.75 & 0.66 & 0.58 & 4.95 & 0.80 & 0.64 \\
\hline SegNet & 0.70 & 15.24 & 0.73 & 0.72 & 0.66 & 7.92 & 0.72 & 0.69 & 0.57 & 4.48 & 0.77 & 0.60 \\
\hline FRRN & 0.71 & 12.66 & 0.75 & 0.73 & 0.71 & 7.72 & 0.69 & 0.71 & 0.60 & 2.91 & 0.76 & 0.64 \\
\hline incremental-MRRN & 0.74 & 7.94 & 0.80 & 0.73 & 0.74 & 5.85 & 0.82 & 0.72 & 0.68 & 2.60 & 0.85 & 0.67 \\
\hline dense-MRRN & 0.73 & 8.10 & 0.79 & 0.73 & 0.73 & 5.94 & 0.80 & 0.72 & 0.67 & 2.72 & 0.82 & 0.70 \\
\hline
\end{tabular}

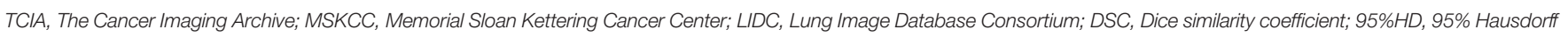
distance; FRRN, full resolution residual neural network; MRRN, multiple resolution residually connected network.

from PET or CT image independently. Each sub-segmentation branch was designed on the basis of the V-Net which is a 3D FCN. Afterwards, two feature maps generated by two parallel branches were fed into the feature fusion module which was comprised of cascaded convolutional operations. In the feature fusion module, high-dimensional information from PET and CT were fused, and re-extracted to generate outputs. They compared the performance of the proposed scheme with scheme utilizing PET or CT only on the same dataset. The comparison of three schemes on DSC were as follows: (PET\&CT: $0.85 \pm 0.08$; PET only: $0.83 \pm 0.10$; CT only: 0.76 $\pm 0.07)$.

In terms of lung cancer, deep learning algorithms proposed in various works have outperformed the existing solutions in most scenarios. However, most recent studies predominantly focused on the segmentation of the GTV, and few studies have explored the usage of this state-of-the-art technique for clinical target volume (CTV) segmentation. Bi et al. (84) established a deep dilated residual network based on ResNet-101 to automatically delineate the CTV for non-small cell lung cancer patients receiving postoperative RT. They summarized that with the assistance of dilated residual network, moderate segmentation accuracy was obtained for the CTV with a DSC of $0.75 \pm 0.06$. It is more challenging to segment the CTV perhaps owing to the following reasons. The postoperative CTV cannot be easily recognized by discriminating tissue density as it was for the GTV because the CTV usually contains the high-risk nodal regions and bronchial stump. Moreover, postoperative changes, for instance blurred soft tissue boundary, ectopic target due to diverse lobectomies, and a wide variety of different patient's lung volume, possibly increase anatomical diversity. Besides, the definition of the CTV is more complex compared with organs. Inter-observer variability resulting from different practical experiences and clinical guidelines has been considered to be a huge challenge in the automated CTV delineating. More information about the aforementioned works is summarized in Table 8.

\section{DISCUSSION}

\section{Comparison Between the Atlas-Based and Deep Learning Based Automatic Segmentation}

Currently, the atlas-based automatic segmentation technique is most commonly employed in clinical practice. The atlas-based automatic segmentation utilizes a reference image as an atlas, in which the boundaries of interested organs are already precisely delineated. The reference image and the new image to be segmented are registered, and the optimal transformation parameters between the two images are obtained. Then the new test image is automatically segmented by propagating the label in the atlas segmentation onto the new test image based on the obtained transformation parameters (85-87). This method has a precise segmentation result in theory, but in practice the segmentation accuracy relies heavily on the similarity between the reference image and the image to be segmented. Additionally, the choice of the deformable image registration algorithm plays a vital role in the performance of segmentation. Due to organ morphology, variety of the individual patient, and image artifacts, accurate image registration is not always ensured. While this issue may be mitigated with a larger and more diverse atlas dataset, it is difficult to contain all potential patterns in the templates given the unpredictability of tumor shape. Besides, accurate image registration is costly in computation, and a large number of atlas templates make the computation cost soaring with the increase in the segmentation accuracy $(38,88-90)$.

Several studies compared the performance of automatic segmentation using atlas-based and deep learning based techniques separately to delineate the OARs in lung cancer, as shown in Table 9. Lustberg et al. (91) compared user adjusted contours after an atlas-based and deep learning based delineation, against manual contours. In terms of the time saved, they reported that the total median was $7.8 \mathrm{~min}$ and $10 \mathrm{~min}$ for using atlas-based and deep learning based contouring software, respectively. With regard to the esophagus, deep learning based contouring software outperformed the atlas-based contouring software with time saved $1.5 \mathrm{~min} v$ s. $0.3 \mathrm{~min}$. Zhu et al. (59) compared atlas-based with deep CNN-based techniques in aspects of automatic segmentation for multiple OARs, using DSC and MSD as evaluation metrics. In respect of the heart, lungs and liver, there was no significant difference between the atlas-based and the deep CNN-based techniques. As for the spinal cord and the esophagus, the deep $\mathrm{CNN}$-based technique had a superior performance than the atlasbased technique (DSC: 0.71 vs. 0.54 \& MSD: $2.6 \mathrm{~mm} v s .3 .1 \mathrm{~mm}$ for the esophagus; DSC: 0.79 vs. $0.71 \&$ MSD: $1.2 \mathrm{~mm}$ vs. $2.2 \mathrm{~mm}$ for the spinal cord). Zhang et al. (64) also compared the CNN-based and atlas-based automatic segmentation techniques. In their study, the CNN-based method performed better than the atlas-based 
TABLE 8 | Selected works on deep learning-based automated segmentation of lung tumors.

\begin{tabular}{|c|c|c|c|c|c|c|c|}
\hline Reference & Year & Network & Datasets & Input & Targets & Results & Research Highlight \\
\hline Wang et al. (36) & 2018 & CNN & 9 patients & MRI & GTV & $\begin{array}{l}\text { DSC: } \\
0.82 \pm 0.10 \\
\text { Precision: } \\
0.81 \pm 0.10\end{array}$ & $\begin{array}{l}\text { establishing a patient-specific adaptive patch-based } \\
\mathrm{CNN} \text { and a population-based CNN, and comparing their } \\
\text { performance with each other }\end{array}$ \\
\hline Zhang et al. (79) & 2020 & ResNet & $\begin{array}{l}330 \text { patients (training set: } \\
300 \text {; test set: } 30)\end{array}$ & $\mathrm{CT}$ & GTV & $\begin{array}{c}\text { DSC: } \\
0.73 \pm 0.07 \\
\text { JSC: } \\
0.68 \pm 0.09 \\
\text { TPR: } \\
0.74 \pm 0.07 \\
\text { FPR: } \\
0.0012 \pm 0.0014\end{array}$ & $\begin{array}{l}\text { proposing a modified version of ResNet to segment the } \\
\text { GTV for NSCLC patients and comparing its performance } \\
\text { with U-net }\end{array}$ \\
\hline Zhao et al. (82) & 2018 & 3D FCN & $\begin{array}{l}84 \text { patients (training set: } 48 \text {; } \\
\text { test set:36) }\end{array}$ & PET/CT & $\begin{array}{l}\text { Lung } \\
\text { tumor }\end{array}$ & $\begin{array}{c}\text { DSC: } \\
0.85 \pm 0.08\end{array}$ & $\begin{array}{l}\text { proposing a multi-modality co-segmentation network and } \\
\text { comparing its performance with utilizing CT or PET only }\end{array}$ \\
\hline Jiang et al. (41) & 2019 & MRRN & $\begin{array}{l}1210 \text { patients from three } \\
\text { datasets ( } 377 \text { from TCIA for } \\
\text { training, } 304 \text { from MSKCC } \\
\text { for validating, and } 529 \text { from } \\
\text { LIDC for testing) }\end{array}$ & $\mathrm{CT}$ & $\begin{array}{l}\text { Lung } \\
\text { tumor }\end{array}$ & $\begin{array}{c}\text { form: } \\
\text { (TCIA, MSKCC, LIDC) } \\
\text { DSC: } \\
\text { (0.74, 0.75, 0.68) } \\
\text { Precision: } \\
\text { (0.73, 0.72, 0.67) } \\
\text { Sensitivity: } \\
\text { (0.80, 0.82, 0.85) } \\
\text { 95\%HD (mm): } \\
(7.94,5.85,2.60)\end{array}$ & $\begin{array}{l}\text { developing two multiple resolution residually connected } \\
\text { network viz. incremental-MRRN and dense-MRRN, and } \\
\text { comparing their performance with other commonly used } \\
\text { networks }\end{array}$ \\
\hline Bi et al. (84) & 2019 & $\begin{array}{l}\text { ResNet- } \\
101\end{array}$ & $\begin{array}{l}269 \text { patients (training set: } \\
\text { 200; validation set: } 50 ; \\
\text { test set: } 19 \text { ) }\end{array}$ & $\mathrm{CT}$ & CTV & $\begin{array}{c}\text { DSC: } \\
0.75 \pm 0.06 \\
\text { MDTA: } \\
2.97 \pm 0.91 \\
\text { CV: } \\
0.129 \pm 0.040 \\
\text { SDD: } \\
0.47 \pm 0.22\end{array}$ & $\begin{array}{l}\text { introducing a deep residual network with dilated blocks } \\
\text { to segment the CTV for NSCLC patients, and comparing } \\
\text { with manual delineation }\end{array}$ \\
\hline
\end{tabular}

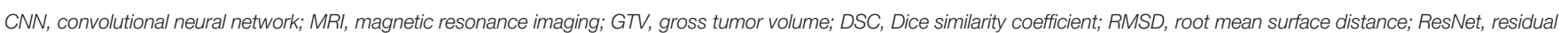

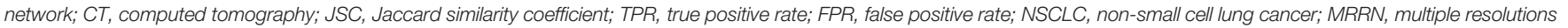

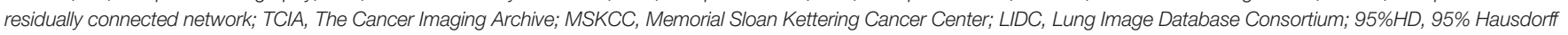
distance; CTV, clinical target volume; MDTA, mean distance to agreement; CV, coefficient of variation; SDD, standard distance deviation.

method in the left lung (DSC: 0.948 vs. 0.932; MSD: $1.10 \mathrm{~mm}$ vs. $1.73 \mathrm{~mm}$ ), heart (DSC: 0.893 vs. 0.858; MSD: $1.65 \mathrm{~mm}$ vs. $3.66 \mathrm{~mm}$ ) and liver (DSC: 0.937 vs. 0.936; MSD: $2.03 \mathrm{~mm}$ vs. $2.11 \mathrm{~mm}$ ). The CNN-based method segmented the esophagus with a mean DSC of 0.732 and an average MSD of $1.38 \mathrm{~mm}$, whereas that of the atlasbased method for the esophagus was unavailable.

Considering the comparison results mentioned above, we can summarize that deep learning based automatic segmentation is more accurate and efficient in delineating the multiple OARs in lung cancer. Although it is not yet available to directly apply a deep learning based algorithm to clinical segmentation for OARs in lung cancer RT, we can use the segmentation generated from it as a starting point and manually adjust it to meet clinical guidelines. Overall, deep learning based automatic segmentation can potentially be employed in clinical routines to relieve radiation oncologists of the tedious contouring work.

\section{CNN, U-Net, and GAN}

With the processing of the CNN layers, the level of abstraction of the extracted features gradually increases. Shallower layers grasp local features, while deeper layers capture global features by using convolution kernels whose receptive fields are much broader. Generally speaking, the deeper CNNs can solve more complex issues. However, with the network depth increasing, the degradation phenomenon has been reported. Surprisingly, such degradation phenomenon is not due to overfitting. Besides, continuously adding more layers to a suitable deep network results in bigger training error. Finally, even though the deeper networks perform better, they are difficult to train owing to the gradient vanishing problem. These reveal that not all CNNs are easy to optimize and the network depth is of crucial importance. It is tedious to find the optimal network depth by trial and error. Introducing residual connections to create shortcuts among blocks of layers may be beneficial to achieve fast and stable training. Nevertheless, when the number of layers in CNNs was rather small, the residual connections may not only fail to help reduce the training difficulty, but also decrease the complexity of the network and thus the expressive power. It is worthy of considering whether or not to add residual connections while designing the network architecture.

$\mathrm{U}-\mathrm{Net}$ is one of the most popular medical image segmentation networks (46). It is possible to train the U-Net to produce precise segmentations with very little labeled training data. Both convolutions and down-sampling operations are 
TABLE 9 | Selected works on comparison between atlas-based and deep learning-based automated segmentation.

\begin{tabular}{|c|c|c|c|c|}
\hline \multirow[t]{2}{*}{ Reference } & \multirow[t]{2}{*}{ Year } & \multirow[t]{2}{*}{ Evaluation Metrics } & \multicolumn{2}{|c|}{ Comparison Results } \\
\hline & & & atlas-based & deep learning-based \\
\hline Lustberg et al. (91) & 2017 & $\begin{array}{l}\text { time saved } \\
\text { (compared with manual segmentation) }\end{array}$ & $7.8 \mathrm{~min}$ & $10 \mathrm{~min}$ \\
\hline Zhu et al. (59) & 2018 & $\begin{array}{l}\mathrm{MSD}(\mathrm{mm}) \\
95 \% \mathrm{HD}(\mathrm{mm})\end{array}$ & $\begin{array}{l}\text { heart: } 0.90 \pm 0.04 \\
\text { liver: } 0.87 \pm 0.05 \\
\text { esophagus: } 0.54 \pm 0.08 \\
\text { spinal cord: } 0.71 \pm 0.06 \\
\text { lungs: } 0.95 \pm 0.01 \\
\text { heart: } 3.14 \pm 1.31 \\
\text { liver: } 3.83 \pm 1.74 \\
\text { esophagus: } 2.67 \pm 1.26 \\
\text { spinal cord: } 3.03 \pm 1.57 \\
\text { lungs: } 1.85 \pm 0.53 \\
\text { heart: } 9.53 \pm 4.99 \\
\text { liver: } 11.87 \pm 5.06 \\
\text { esophagus: } 9.45 \pm 4.64 \\
\text { spinal cord: } 11.97 \pm 6.88 \\
\text { lungs: } 8.07 \pm 2.39\end{array}$ & $\begin{array}{l}\text { heart: } 0.91 \pm 0.03 \\
\text { liver: } 0.89 \pm 0.02 \\
\text { esophagus: } 0.71 \pm 0.05 \\
\text { spinal cord: } 0.79 \pm 0.03 \\
\text { lungs: } 0.95 \pm 0.01 \\
\text { heart: } 2.92 \pm 1.51 \\
\text { liver: } 3.21 \pm 0.93 \\
\text { esophagus: } 2.18 \pm 0.80 \\
\text { spinal cord: } 1.25 \pm 0.23 \\
\text { lungs: } 1.93 \pm 0.51 \\
\text { heart: } 7.98 \pm 4.56 \\
\text { liver: } 10.06 \pm 4.28 \\
\text { esophagus: } 7.83 \pm 2.85 \\
\text { spinal cord: } 4.01 \pm 2.05 \\
\text { lungs: } 7.96 \pm 2.57\end{array}$ \\
\hline Zhang et al. (64) & 2020 & $\begin{array}{l}\text { average time } \\
\text { DSC } \\
\text { MSD (mm) }\end{array}$ & $\begin{array}{l}2.4 \text { minutes } \\
\text { left lung: } 0.932 \pm 0.040 \\
\text { right lung: } 0.943 \pm 0.017 \\
\text { heart: } 0.858 \pm 0.077 \\
\text { spinal cord: } 0.868 \pm 0.031 \\
\text { liver:0.936 } \pm 0.012 \\
\text { esophagus: }- \\
\text { left lung: } 1.73 \pm 1.58 \\
\text { right lung: } 2.17 \pm 2.44 \\
\text { heart: } 3.66 \pm 2.44 \\
\text { spinal cord: } 0.66 \pm 0.16 \\
\text { liver: } 2.11 \pm 1.31 \\
\text { esophagus: }-\end{array}$ & $\begin{array}{l}1.6 \text { minutes } \\
\text { left lung: } 0.948 \pm 0.013 \\
\text { right lung: } 0.943 \pm 0.015 \\
\text { heart: } 0.893 \pm 0.048 \\
\text { spinal cord: } 0.821 \pm 0.046 \\
\text { liver: } 0.937 \pm 0.027 \\
\text { esophagus: } 0.732 \pm 0.069 \\
\text { left lung: } 1.10 \pm 0.15 \\
\text { right lung: } 2.23 \pm 2.33 \\
\text { heart: } 1.65 \pm 0.48 \\
\text { spinal cord: } 0.87 \pm 0.21 \\
\text { liver: } 2.03 \pm 1.49 \\
\text { esophagus: } 1.38 \pm 0.44\end{array}$ \\
\hline
\end{tabular}

DSC, Dice similarity coefficient; MSD, mean surface distance; 95\%HD, 95\% Hausdorff distance.

usually local operations, meaning that a lot of local operators need to be stacked in a cascade way to aggregate long-range information (92). Meanwhile, the amount of training parameters also increases while stacking them, which becomes a large obstacle to improve the calculation efficiency. Additionally, more down-sampling operations lead to the loss of more spatial information during encoding, resulting in poor accuracy of medical image segmentation. Of course, those issues exist in the decoder as well. In allusion to this instance, some researchers attempt to address those limitations. Wang et al. successfully solved this issue by proposing a novel network architecture called non-local U-Net, which is equipped with flexible global aggregation blocks based on the self-attention operators (92-95).

GAN, as a method of unsupervised deep learning, is commonly used in medical image segmentation. GAN can train any kind of network architecture as its generator network. GAN dispenses with the need for using Markov chain to sample repeatedly, and for inferencing in the learning process, which avoids the difficulty of calculating the probability. But there are some challenges faced by GAN, such as how to break through the non-convergence problem and collapse problem (96) which may occur in the learning process.

\section{Current Challenges and Limitations}

With the significant progress in computer science and techniques, deep learning algorithms play an indispensable role in image segmentation with their compelling ability to extract features automatically. According to studies in recent years, image segmentation technologies based on deep learning have surpassed traditional segmentation methods in segmentation efficiency and accuracy. Nonetheless, deep learning based automatic segmentation techniques still face various challenges and limitations.

\section{Low Contrast Issue}

Unlike natural images, the information contained in medical images is more complicated, and the similarity between the target and the surrounding background in the image is extremely high for low contrast tissues. Therefore, it is difficult to accurately detect target boundaries or to delineate the target from the background. Besides, to guarantee the RT outcomes, high accuracy is required. So, the first problem to solve is that how to precisely identify the boundaries of low contrast tissues such as esophagus etc. and delineate them with superior performance. Currently, proposed deep learning based automatic segmentation algorithms merely managed to 
segment high contrast organs in CT with satisfactory results, and usually failed to segment the esophagus with high accuracy. This inferior performance may be explained by the following reasons. First, the appearance of esophagus varies depending on whether it is full of air, of remains of orally given contrast agent, or both (97). Second, the esophagus has certain mobility, which leads to the fact that the esophagus has a greatly inhomogeneous appearance and a versatile shape. Furthermore, studies have indicated that owing to respiration and cardiac motion, esophageal intrafraction motion is generally between 5 and $10 \mathrm{~mm}$ and can reach up to $15 \mathrm{~mm}$ (98100). How to improve the inferior segmentation accuracy of the esophagus will become one of the key research directions. MRI has a superior visualization of low contrast tissues compared to CT. Perhaps using MRI data as input could improve the segmentation accuracy to a certain extent. Nevertheless, we ought to put more emphasis on improving existing deep learning segmentation algorithms or coming up with novel ones. Given that 3D-based convolutions could address volumetric homogeneity better and take full advantage of the 3D spatial context compared with 2Dbased convolutions, it may be beneficial to use a $3 \mathrm{D}$ network to segment the esophagus which has a thin tubular and continuous structure. Fechter et al. (97) have proposed a new scheme and achieved competitive results. Firstly, they employed a 3D-FNN to yield a first estimation of the esophagus. Then, an active contour model and a random walker approach were used to refine the first estimation to the final contour.

\section{Size of Dataset}

The size of the training dataset greatly affects the robustness of deep learning algorithms. It could be argued that the generalization of deep learning algorithms is expected to increase with an enlarged training dataset. Currently, most studies use different datasets collected individually, except in some segmentation competitions. Besides, most of the datasets reported in this review are not adequate in the era of big data. Taking a limited dataset for training and testing may lead to model over-fitting. Hence the efficient generalization of the proposed algorithms cannot be demonstrated. Utilizing a transfer learning strategy may potentially handle the issue of limited data size. Image-Net is typically employed for pretraining networks to process medical images (101). On the other hand, data augmentation is also an effective method to address the issue of a limited dataset. Furthermore, it is worth considering of establishing a public image dataset with a highquality ground truth label to make advances in deep learning based automatic segmentation techniques.

However, it is not practical to establish an adequate public image dataset for the initial training of the deep learning model within a short time. At present, the amount of data utilized for the initial training is most likely not adequate. With the accumulation of clinical cases, we can utilize new cases to further fine-tune the deep learning model to achieve better performance. Notably, catastrophic forgetting may occur during the fine-tuning process. While we can solve this issue by retraining the deep learning model utilizing both old and new cases, this approach is tedious and inefficient. Moreover, manual labeling the new cases is also a time-consuming and laborious task. In view of such a situation, Men et al. (102) proposed a novel scheme. In addition to training an automatic segmentation network, they also trained a binary classifier to judge the quality of the automatic segmentation. For a batch of new clinical cases, the segmentation network firstly performs the automatic segmentation. Then, the binary classifier judges the segmentation result and selects the case with a DSC less than the setting threshold. These selected cases are manually labeled by radiotherapy experts and then used to fine-tune the segmentation network to improve its performance. Their scheme remarkably reduces the manual labeling effort and enables the deep learning model to continually update over the accumulation of clinical cases, thus achieving the strategy of continual learning. Their method could be explored to efficiently improve the robustness of deep learning models in the future.

\section{Lack of Consensus on Guidelines}

Another limitation of deep learning based automatic segmentation technique is that we usually cannot objectively determine whether a clinically acceptable ground truth is an optimal case due to lack of consensus. In general, the shape and position of organs vary greatly among different patients due to race, gender, age and progression of the disease etc. Radiation oncologists manually segment the OARs and GTV to generate the ground truth depending on their own prior knowledge and experience, which leads to inconsistencies in process of generating the ground truth for both inter- and intraobservers. Ground truth plays a vital role in the performance of deep learning algorithms. Moreover, differences in image acquisition protocols (such as posture and breath-hold conditions etc.) could also potentially affect the performance of deep learning algorithms. Besides, it is also necessary to establish international consensus on guidelines to eliminate the inconsistencies that existed in contouring the ground truth for both inter- and intra-observers.

\section{Network Design}

With the increase of the number of layers used in the network, the deep learning algorithm has stronger feature expressive power, making subsequent predictions easier and more accurate. However, the complexity of the deep learning algorithm also increases simultaneously, which means that the network training must take more time and GPU memory. Furthermore, to extract and integrate multi-scale features, most existing methods attempt to propose and add more complex blocks and strategies to commonly used networks, which significantly increases the GPU memory and computation cost. So, it is worthwhile to think about how to achieve a balance between network design and computation time or cost. In this review, the highest accuracy reported in terms of DSC was achieved by $\mathrm{Hu}$ et al. (65) for the lung segmentation. They combined the Mask R-CNN with the K-means kernel to achieve accurate segmentation. With regard to OARs, Han et al. (40) developed a multi-resolution VB-Net architecture and achieved the best performance in segmentation of heart, aorta, trachea, esophagus. Moreover, it is worthwhile to explore to stack networks sequentially to build cascaded architectures or to build multi-level nested architectures. Zhang et al. (103) designed a slice classification model-facilitated $3 \mathrm{D}$ encoder-decoder network for segmenting OARs in head and neck cancer. The utilization of the 
slice classification model alleviates class-imbalance issues existing in small volume OARs, and decreases unnecessary computation time. Qin et al. (104) proposed a two-level nested U-Net structure called $\mathrm{U}^{2}$-Net for salient object detection and obtained competitive performance against other state-of-the-art networks at a low GPU memory and computation cost. The researchers who are interested in this domain can grope for novel network architectures so that the performance of segmentation can be improved.

\section{Clinical Issues}

Deep learning algorithms are referred to as black-box algorithms owing to lack of interpretability. It is hard to fully understand how, and which factors result in poor segmentation performance. Namely, the deep learning algorithm may fail to segment the OARs and GTV in an unpredictable way which is dangerous in clinical practice. Before deep learning based segmentation techniques can be made clinically available, we ought to consider the legal and ethical responsibilities, and the issues of ensuing patient safety. Therefore, it is of vital importance to implement an exhaustive, comprehensive, and rigid quality assurance procedure for deep learning based segmentation techniques to assure adequately high accuracy of the segmentation with complete conformance to a set of safety criteria. Independent scoring software and commercial thirdparty assessment software may possibly serve as tools to handle the issues originating from automated segmentation algorithms. Lastly, it is also important to guarantee that all generated segmentations information can be exported/imported consistently and accurately to other systems such as the treatment planning system. The limitation of the automatic segmentation techniques should be stated so that the users are aware and vendors can address these issues (105).

\section{SUMMARY}

Deep learning based automatic segmentation techniques have rapidly become the state-of-the-art technique in delineating the OARs and GTV in lung cancer RT. The auto-segmentation of lung, heart and liver has achieved satisfactory results. However,

\section{REFERENCES}

1. Sung H, Ferlay J, Siegel RL, Laversanne M, Soerjomataram I, Jemal A, et al. Global Cancer Statistics 2020: GLOBOCAN Estimates of Incidence and Mortality Worldwide for 36 Cancers in 185 Countries. CA Cancer J Clin (2021) 71(3):209-49. doi: 10.3322/caac.21660

2. Chen Z, Zhang P, Xu Y, Yan J, Liu Z, Lau WB, et al. Surgical Stress and Cancer Progression: The Twisted Tango. Mol Cancer (2019) 18(1):132. doi: 10.1186/s12943-019-1058-3

3. Hoffmann H, Passlick B, Ukena D, Wesselmann S. Surgical Therapy for Lung Cancer: Why It Should be Performed in High Volume Centres. Pneumologie (Stuttgart Germany) (2020) 74(10):670-7. doi: 10.1055/a1172-5675

4. Pirker R. Chemotherapy Remains a Cornerstone in the Treatment of Nonsmall Cell Lung Cancer. Curr Opin Oncol (2020) 32(1):63-7. doi: $10.1097 /$ cco.0000000000000592 one still has to study how to improve the segmentation performance of esophagus taking into account low contrast and respiration motion and other factors. When it comes to segmentation of the GTV, the studies are rather few thus far and the segmentation performance is poor. We still need to make effort to improve accuracy in delineating the GTV and even the CTV. Deep learning based automatic segmentation is a rapidly developing field. Over the next few years, a further modification of deep learning algorithms may be explored to address the remaining issues and improve the accuracy of segmentation. It holds great promise to employ a deep learning based technique as a highly useful tool to automatically segment the OARs and GTV for routine clinical use under expert visual inspection and approval. The promising result of segmentation potentially contributes to optimizing RT planning and developing adaptive radiotherapy. Finally, cautions must be taken in terms of all aspects of limitations before deep learning based automatic segmentation is used for clinical practice.

\section{AUTHOR CONTRIBUTIONS}

XL and RY wrote the manuscript. K-WL and L-SG helped with manuscript redaction. RY carried out a technical review of the manuscript in aspects of clinical practice and deep learning, and revised the manuscript. All authors contributed to the article and approved the submitted version.

\section{FUNDING}

This work is partly supported by the National Natural Science Foundation of China under Grants Nos.11735003, 11975041, and 11961141004, the fundamental Research Funds for the Central Universities, National Key Research and Development Program of China (2021YFE0202500), Capital's Funds for Health Improvement and Research (2020-2Z-40919), Beijing Municipal Commission of Science and Technology Collaborative Innovation Project (Z201100005620012), and China International Medical Foundation, HDRS2020030206.
5. Allen C, Her S, Jaffray DA. Radiotherapy for Cancer: Present and Future. Adv Drug Deliv Rev (2017) 109:1-2. doi: 10.1016/j.addr.2017.01.004

6. Brown S, Banfill K, Aznar MC, Whitehurst P, Faivre Finn C. The Evolving Role of Radiotherapy in Non-Small Cell Lung Cancer. Br J Radiol (2019) 92 (1104):20190524. doi: 10.1259/bjr.20190524

7. Baker S, Dahele M, Lagerwaard FJ, Senan S. A Critical Review of Recent Developments in Radiotherapy for Non-Small Cell Lung Cancer. Radiat Oncol (London England) (2016) 11(1):115. doi: 10.1186/s13014-016-0693-8

8. Habash RWY. Therapeutic Hyperthermia. Handb Clin Neurol (2018) 157:853-68. doi: 10.1016/b978-0-444-64074-1.00053-7

9. Hurwitz MD. Hyperthermia and Immunotherapy: Clinical Opportunities. Int $J$ Hyperthermia: Off J Eur Soc Hyperthermic Oncol North Am Hyperthermia Group (2019) 36(sup1):4-9. doi: 10.1080/02656736.2019.1653499

10. Gou Q, Zhou Z, Zhao M, Chen X, Zhou Q. Advances and Challenges of Local Thermal Ablation in Non-Small Cell Lung Cancer. Zhongguo fei ai za zhi =Chin J Lung Cancer (2020) 23(2):111-7. doi: 10.3779/j.issn.1009-3419.2020.02.06 
11. Steven A, Fisher SA, Robinson BW. Immunotherapy for Lung Cancer. Respirol (Carlton Vic) (2016) 21(5):821-33. doi: 10.1111/resp.12789

12. Aldarouish M, Wang C. Trends and Advances in Tumor Immunology and Lung Cancer Immunotherapy. J Exp Clin Cancer Res: CR (2016) 35(1):157. doi: 10.1186/s13046-016-0439-3

13. Burdett S, Rydzewska L, Tierney J, Fisher D, Parmar MK, Arriagada R, et al. Postoperative Radiotherapy for Non-Small Cell Lung Cancer. Cochrane Database Syst Rev (2016) 9(9):Cd002142. doi: 10.1002/14651858. CD002142.pub3

14. Nagata Y, Kimura T. Stereotactic Body Radiotherapy (SBRT) for Stage I Lung Cancer. Jpn J Clin Oncol (2018) 48(5):405-9. doi: 10.1093/jjco/hyy034

15. Hamaji M. Surgery and Stereotactic Body Radiotherapy for Early-Stage Non-Small Cell Lung Cancer: Prospective Clinical Trials of the Past, the Present, and the Future. Gen Thorac Cardiovasc Surg (2020) 68(7):692-6. doi: 10.1007/s11748-019-01239-8

16. Chung SY, Chang JS, Choi MS, Chang Y, Choi BS, Chun J, et al. Clinical Feasibility of Deep Learning-Based Auto-Segmentation of Target Volumes and Organs-at-Risk in Breast Cancer Patients After Breast-Conserving Surgery. Radiat Oncol (London England) (2021) 16(1):44. doi: 10.1186/ s13014-021-01771-z

17. Choi MS, Choi BS, Chung SY, Kim N, Chun J, Kim YB, et al. Clinical Evaluation of Atlas- and Deep Learning-Based Automatic Segmentation of Multiple Organs and Clinical Target Volumes for Breast Cancer. Radiother Oncol: J Eur Soc Ther Radiol Oncol (2020) 153:139-45. doi: 10.1016/ j.radonc.2020.09.045

18. Wong J, Fong A, McVicar N, Smith S, Giambattista J, Wells D, et al. Comparing Deep Learning-Based Auto-Segmentation of Organs at Risk and Clinical Target Volumes to Expert Inter-Observer Variability in Radiotherapy Planning. Radiother Oncol: J Eur Soc Ther Radiol Oncol (2020) 144:152-8. doi: 10.1016/j.radonc.2019.10.019

19. Wang Z, Chang Y, Peng Z, Lv Y, Shi W, Wang F, et al. Evaluation of Deep Learning-Based Auto-Segmentation Algorithms for Delineating Clinical Target Volume and Organs at Risk Involving Data for 125 Cervical Cancer Patients. J Appl Clin Med Phys (2020) 21(12):272-9. doi: 10.1002/ acm2.13097

20. Men K, Dai J, Li Y. Automatic Segmentation of the Clinical Target Volume and Organs at Risk in the Planning CT for Rectal Cancer Using Deep Dilated Convolutional Neural Networks. Med Phys (2017) 44(12):6377-89. doi: $10.1002 / \mathrm{mp} .12602$

21. Vrtovec T, Močnik D, Strojan P, Pernuš F, Ibragimov B. Auto-Segmentation of Organs at Risk for Head and Neck Radiotherapy Planning: From AtlasBased to Deep Learning Methods. Med Phys (2020) 47(9):e929-50. doi: 10.1002/mp.14320

22. Kholiavchenko M, Sirazitdinov I, Kubrak K, Badrutdinova R, Kuleev R, Yuan Y, et al. Contour-Aware Multi-Label Chest X-Ray Organ Segmentation. Int J Comput Assist Radiol Surg (2020) 15(3):425-36. doi: 10.1007/s11548-019-02115-9

23. Yahyatabar M, Jouvet P, Cheriet F. Dense-Unet: A Light Model for Lung Fields Segmentation in Chest X-Ray Images. Annu Int Conf IEEE Eng Med Biol Soc IEEE Eng Med Biol Soc Annu Int Conf (2020) 2020:1242-5. doi: 10.1109/embc44109.2020.9176033

24. Candemir S, Antani S. A Review on Lung Boundary Detection in Chest XRays. Int J Comput Assist Radiol Surg (2019) 14(4):563-76. doi: 10.1007/ s11548-019-01917-1

25. Beveridge JR, Griffith J, Kohler RR, Hanson AR, Riseman EM. Segmenting Images Using Localized Histograms and Region Merging. Int J Comput Vision (1989) 2(3):311-47. doi: 10.1007/BF00158168

26. Pal NR, Pal SK. A Review on Image Segmentation Techniques. Pattern Recognit (1993) 26(9):1277-94. doi: 10.1016/0031-3203(93)90135-J

27. Freund Y, Schapire RE. A Decision-Theoretic Generalization of On-Line Learning and an Application to Boosting. J Comput Syst Sci (1997) 55 (1):119-39. doi: 10.1006/jcss.1997.1504

28. Vo A-V, Truong-Hong L, Laefer DF, Bertolotto M. Octree-Based Region Growing for Point Cloud Segmentation. ISPRS J Photogramm Remote Sens (2015) 104:88-100. doi: 10.1016/j.isprsjprs.2015.01.011

29. Lee LK, Liew SC, Thong WJ. A Review of Image Segmentation Methodologies in Medical Image. In: Advanced Computer and Communication Engineering Technology: 2015//2015. Cham: Springer International Publishing (2015). p. 1069-80.
30. Cabezas M, Oliver A, Lladó X, Freixenet J, Cuadra MB. A Review of AtlasBased Segmentation for Magnetic Resonance Brain Images. Comput Methods Programs Biomed (2011) 104(3):e158-177. doi: 10.1016/ j.cmpb.2011.07.015

31. Wang L, Chitiboi T, Meine H, Günther M, Hahn HK. Principles and Methods for Automatic and Semi-Automatic Tissue Segmentation in MRI Data. Magma (New York NY) (2016) 29(2):95-110. doi: 10.1007/s10334015-0520-5

32. Mansoor A, Bagci U, Foster B, Xu Z, Papadakis GZ, Folio LR, et al. Segmentation and Image Analysis of Abnormal Lungs at CT: Current Approaches, Challenges, and Future Trends. RadioGraphics (2015) 35 (4):1056-76. doi: 10.1148/rg.2015140232

33. Sahiner B, Pezeshk A, Hadjiiski LM, Wang X, Drukker K, Cha KH, et al. Deep Learning in Medical Imaging and Radiation Therapy. Med Phys (2019) 46(1):e1-e36. doi: 10.1002/mp.13264

34. Shaziya H, Shyamala K, Zaheer R. (2018). Automatic Lung Segmentation on Thoracic CT Scans Using U-Net Convolutional Network, In: 2018 International Conference on Communication and Signal Processing (ICCSP), 3-5 April 2018, Vol. 2018. pp. 0643-7. doi: 10.1109/ ICCSP.2018.8524484

35. Zhao T, Gao D, Wang J, Yin Z. (2018). Lung Segmentation in CT Images Using a Fully Convolutional Neural Network With Multi-Instance and Conditional Adversary Loss, In: 2018 IEEE 15th International Symposium on Biomedical Imaging (ISBI 2018), (2018) pp. 505-9. doi: 10.1109/ ISBI.2018.8363626

36. Wang C, Tyagi N, Rimner A, Hu YC, Veeraraghavan H, Li G, et al. Segmenting Lung Tumors on Longitudinal Imaging Studies via a PatientSpecific Adaptive Convolutional Neural Network. Radiother Oncol: J Eur Soc Ther Radiol Oncol (2019) 131:101-7. doi: 10.1016/j.radonc.2018.10.037

37. Abdullah MF, Mansor MS, Sulaiman SN, Osman MK, Marzuki NNSM, Isa IS, et al. (2019). A Comparative Study of Image Segmentation Technique Applied for Lung Cancer Detection, in: 2019 9th IEEE International Conference on Control System, Computing and Engineering (ICCSCE), (2019) pp. 72-7. doi: 10.1109/ICCSCE47578.2019.9068574

38. Dong X, Lei $\mathrm{Y}$, Wang T, Thomas M, Tang L, Curran WJ, et al. Automatic Multiorgan Segmentation in Thorax CT Images Using U-Net-GAN. Med Phys (2019) 46(5):2157-68. doi: 10.1002/mp.13458

39. Feng X, Qing K, Tustison NJ, Meyer CH, Chen Q. Deep Convolutional Neural Network for Segmentation of Thoracic Organs-at-Risk Using Cropped 3D Images. Med Phys (2019) 46(5):2169-80. doi: 10.1002/ mp.13466

40. Han M, Yao G, Zhang W, Mu G, Zhan Y, Zhou X, et al. Segmentation of CT Thoracic Organs by Multiresolution VB-Nets. In: CEUR Workshop Proceedings, (2019). vol. 2349, p. 1-4. Available at: http://ceur-ws.org/Vol2349/SegTHOR2019\_paper\1.pdf

41. Jiang J, Hu YC, Liu CJ, Halpenny D, Hellmann MD, Deasy JO, et al. Multiple Resolution Residually Connected Feature Streams for Automatic Lung Tumor Segmentation From CT Images. IEEE Trans Med Imaging (2019) 38(1):134-44. doi: 10.1109/TMI.2018.2857800

42. Portela RDS, Pereira JRG, Costa MGF, Filho CFFC. (2020). Lung Region Segmentation in Chest X-Ray Images Using Deep Convolutional Neural Networks, In: 2020 42nd Annual International Conference of the IEEE Engineering in Medicine \& Biology Society (EMBC), 20-24 July 2020, Vol. 2020. pp. 1246-9. doi: 10.1109/EMBC44109.2020.9175478

43. Avanzo M, Stancanello J, Pirrone G, Sartor G. Radiomics and Deep Learning in Lung Cancer. Strahlenther Onkol: Organ der Deutschen Rontgengesellschaft [et al] (2020) 196(10):879-87. doi: 10.1007/s00066-020-01625-9

44. LeCun Y, Kavukcuoglu K, Farabet C. (2010). Convolutional Networks and Applications in Vision, in: Proceedings of 2010 IEEE International Symposium on Circuits and Systems, (2010) pp. 253-6. doi: 10.1109/ ISCAS.2010.5537907

45. Long J, Shelhamer E, Darrell T. (2015) Fully Convolutional Networks for Semantic Segmentation. 2015 IEEE Conference on Computer Vision and Pattern Recognition (CVPR), (2015) pp. 3431-40. doi: 10.1109/ CVPR.2015.7298965

46. Ronneberger O, Fischer P, Brox T. (2015) U-Net: Convolutional Networks for Biomedical Image Segmentation. In: N Navab, J Hornegger, W Wells, A Frangi. (eds) Medical Image Computing and Computer-Assisted Intervention 
- MICCAI 2015. MICCAI 2015. Lecture Notes in Computer Science, vol 9351. Springer, Cham. doi: 10.1007/978-3-319-24574-4_28

47. Siegel RL, Miller KD, Jemal A. Cancer Statistics, 2020. CA: A Cancer J Clin (2020) 70(1):7-30. doi: 10.3322/caac.21590

48. Sheng K. Artificial Intelligence in Radiotherapy: A Technological Review. Front Med (2020) 14(4):431-49. doi: 10.1007/s11684-020-0761-1

49. Men K, Zhang T, Chen X, Chen B, Tang Y, Wang S, et al. Fully Automatic and Robust Segmentation of the Clinical Target Volume for Radiotherapy of Breast Cancer Using Big Data and Deep Learning. Phys Med (2018) 50:13-9. doi: 10.1016/j.ejmp.2018.05.006

50. Liu C, Gardner SJ, Wen N, Elshaikh MA, Siddiqui F, Movsas B, et al. Automatic Segmentation of the Prostate on CT Images Using Deep Neural Networks (DNN). Int J Radiat Oncol Biol Phys (2019) 104(4):924-32. doi: 10.1016/j.jijrobp.2019.03.017

51. Yang Q, Zhang S, Sun X, Sun J, Yuan K. (2019). Automatic Segmentation of Head-Neck Organs by Multi-Mode CNNs for Radiation Therapy, in: 2019 International Conference on Medical Imaging Physics and Engineering (ICMIPE), (2019) p. 1-5. doi: 10.1109/ICMIPE47306.2019.9098166

52. Alkassar S, Abdullah MAM, Jebur BA. (2019). Automatic Brain Tumour Segmentation Using Fully Convolution Network and Transfer Learning, In: 2019 2nd International Conference on Electrical, Communication, Computer, Power and Control Engineering (ICECCPCE), (2019) 188-92. doi: 10.1109/ ICECCPCE46549.2019.203771

53. Mathews C, Mohamed A. (2020). Review of Automatic Segmentation of MRI Based Brain Tumour Using U-Net Architecture, In: 2020 Fourth International Conference on Inventive Systems and Control (ICISC), (2020) p. 46-50. doi: 10.1109/ICISC47916.2020.9171057

54. Mesbahi S, Yazid H. (2020). Automatic Segmentation of Medical Images Using Convolutional Neural Networks, In: 2020 5th International Conference on Advanced Technologies for Signal and Image Processing (ATSIP), (2020) pp. 1-5. doi: 10.1109/ATSIP49331.2020.9231669

55. Bhuvaneswari M. (2021). Automatic Segmenting Technique of Brain Tumors With Convolutional Neural Networks in MRI Images, in: 2021 6th International Conference on Inventive Computation Technologies (ICICT), (2021) pp. 759-64. doi: 10.1109/ICICT50816.2021.9358737

56. Li Y, Zhao G, Zhang Q, Lin Y, Wang M. SAP-cGAN: Adversarial Learning for Breast Mass Segmentation in Digital Mammogram Based on Superpixel Average Pooling. Med Phys (2021) 48(3):1157-67. doi: 10.1002/mp.14671

57. Akila Agnes S, Anitha J, Dinesh Peter J. Automatic Lung Segmentation in Low-Dose Chest CT Scans Using Convolutional Deep and Wide Network (CDWN). Neural Comput Appl (2020) 32(20):15845-55. doi: 10.1007/ s00521-018-3877-3

58. Armato Iii SG, McLennan G, Bidaut L, McNitt-Gray MF, Meyer CR, Reeves AP, et al. The Lung Image Database Consortium (LIDC) and Image Database Resource Initiative (IDRI): A Completed Reference Database of Lung Nodules on CT Scans. Med Phys (2011) 38(2):915-31. doi: 10.1118/1.3528204

59. Zhu J, Zhang J, Qiu B, Liu Y, Liu X, Chen L. Comparison of the Automatic Segmentation of Multiple Organs at Risk in CT Images of Lung Cancer Between Deep Convolutional Neural Network-Based and Atlas-Based Techniques. Acta Oncol (2019) 58(2):257-64. doi: 10.1080/ 0284186X.2018.1529421

60. Lambert Z, Petitjean C, Dubray B, Ruan S. SegTHOR: Segmentation of Thoracic Organs at Risk in CT images, 2020 Tenth International Conference on Image Processing Theory, Tools and Applications (IPTA), (2020) p. 1-6, doi: 10.1109/IPTA50016.2020.9286453

61. van Harten LD, Noothout JMH, Verhoeff JJC, Wolterink JM, Išgum I . Automatic Segmentation of Organs at Risk in Thoracic Ct Scans by Combining 2D and 3D Convolutional Neural Networks. In: SegTHOR@ ISBI. in CEUR Workshop Proceedings (2019). vol. 2349, p. 1-4. http://ceurws.org/Vol-2349/SegTHOR2019_paper_12.pdf

62. He T, Hu J, Song Y, Guo J, Yi Z. Multi-Task Learning for the Segmentation of Organs at Risk With Label Dependence. Med Image Anal (2020) 61:101666. doi: 10.1016/j.media.2020.101666

63. Vesal S, Ravikumar N, Maier A. A 2D Dilated Residual U-Net for MultiOrgan Segmentation in Thoracic CT. (2019). vol. 2349, p. 1-4. Available at: http://ceur-ws.org/Vol-2349/SegTHOR2019_paper_13.pdf

64. Zhang T, Yang Y, Wang J, Men K, Wang X, Deng L, et al. Comparison Between Atlas and Convolutional Neural Network Based Automatic
Segmentation of Multiple Organs at Risk in Non-Small Cell Lung Cancer. Med (Baltimore) (2020) 99(34):e21800. doi: 10.1097/MD.0000000000021800

65. Hu Q, de FSLF, Holanda GB, Alves SSA, Dos SSFH, Han T, et al. An Effective Approach for CT Lung Segmentation Using Mask Region-Based Convolutional Neural Networks. Artif Intell Med (2020) 103:101792. doi: 10.1016/j.artmed.2020.101792

66. Tan J, Jing L, Huo Y, Li L, Akin O, Tian Y. LGAN: Lung Segmentation in CT Scans Using Generative Adversarial Network. Comput Med Imaging Graph (2021) 87:101817. doi: 10.1016/j.compmedimag.2020.101817

67. Pawar SP, Talbar SN. LungSeg-Net: Lung Field Segmentation Using Generative Adversarial Network. Biomed Signal Process Control (2021) 64:102296. doi: 10.1016/j.bspc.2020.102296

68. He K, Zhang X, Ren S, Sun J. Deep Residual Learning for Image Recognition. 2016 IEEE Conference on Computer Vision and Pattern Recognition (CVPR), (2016) p. 770-8. doi: 10.1109/CVPR.2016.90

69. Simonyan K, Zisserman A. Very Deep Convolutional Networks for LargeScale Image Recognition. 3rd International Conference on Learning Representations, ICLR 2015, San Diego, 7-9 May 2015 (2015). p. 1-14. Available at: http://arxiv.org/abs/1409.1556

70. Darby SC, Ewertz M, McGale P, Bennet AM, Blom-Goldman U, Brønnum D, et al. Risk of Ischemic Heart Disease in Women After Radiotherapy for Breast Cancer. New Engl J Med (2013) 368(11):987-98. doi: 10.1056/NEJMoa1209825

71. van den Bogaard VAB, Ta BDP, van der Schaaf A, Bouma AB, Middag AMH, Bantema-Joppe EJ, et al. Validation and Modification of a Prediction Model for Acute Cardiac Events in Patients With Breast Cancer Treated With Radiotherapy Based on Three-Dimensional Dose Distributions to Cardiac Substructures. J Clin Oncol (2017) 35(11):1171-8. doi: 10.1200/ JCO.2016.69.8480

72. Vivekanandan S, Landau DB, Counsell N, Warren DR, Khwanda A, Rosen $\mathrm{SD}$, et al. The Impact of Cardiac Radiation Dosimetry on Survival After Radiation Therapy for Non-Small Cell Lung Cancer. Int J Radiat Oncol Biol Phys (2017) 99(1):51-60. doi: 10.1016/j.ijrobp.2017.04.026

73. Yusuf SW, Sami S, Daher IN. Radiation-Induced Heart Disease: A Clinical Update. Cardiol Res Pract (2011) 2011:317659. doi: 10.4061/2011/317659

74. Patel SA, Mahmood S, Nguyen T, Yeap BY, Jimenez RB, Meyersohn NM, et al. Comparing Whole Heart Versus Coronary Artery Dosimetry in Predicting the Risk of Cardiac Toxicity Following Breast Radiation Therapy. Int J Radiat Oncol Biol Phys (2018) 102(3):S46. doi: 10.1016/j.jirobp.2018.06.091

75. Morris ED, Ghanem AI, Dong M, Pantelic MV, Walker EM, Glide-Hurst CK. Cardiac Substructure Segmentation With Deep Learning for Improved Cardiac Sparing. Med Phys (2020) 47(2):576-86. doi: 10.1002/mp.13940

76. McCollough CH, Leng S, Yu L, Fletcher JG. Dual- and Multi-Energy CT: Principles, Technical Approaches, and Clinical Applications. Radiology (2015) 276(3):637-53. doi: 10.1148/radiol.2015142631

77. Chen S, Roth H, Dorn S, May M, Cavallaro A, Lell MM, et al. Towards Automatic Abdominal Multi-Organ Segmentation in Dual Energy CT Using Cascaded 3d Fully Convolutional Network. arXiv e-prints (2017). arXiv:1710.05379. arXiv:1710.05379.

78. Chen S, Zhong X, Hu S, Dorn S, Kachelrieß M, Lell M, et al. Automatic Multi-Organ Segmentation in Dual-Energy CT (DECT) With Dedicated 3D Fully Convolutional DECT Networks. Med Phys (2020) 47(2):552-62. doi: $10.1002 / \mathrm{mp} .13950$

79. Zhang F, Wang Q, Li H. Automatic Segmentation of the Gross Target Volume in Non-Small Cell Lung Cancer Using a Modified Version of ResNet. Technol Cancer Res Treat (2020) 19:1533033820947484. doi: 10.1177/1533033820947484

80. Pohlen T, Hermans A, Mathias M, Leibe B. Full-Resolution Residual Networks for Semantic Segmentation in Street Scenes, 2017 IEEE Conference on Computer Vision and Pattern Recognition (CVPR).. (2017), pp. 3309-18, doi: 10.1109/CVPR.2017.353

81. Badrinarayanan V, Kendall A, Cipolla R. SegNet: A Deep Convolutional Encoder-Decoder Architecture for Image Segmentation. IEEE Trans Pattern Anal Mach Intell (2017) 39(12):2481-95. doi: 10.1109/TPAMI.2016.2644615

82. Zhao X, Li L, Lu W, Tan S. Tumor Co-Segmentation in PET/CT Using Multi-Modality Fully Convolutional Neural Network. Phys Med Biol (2018) 64(1):015011. doi: 10.1088/1361-6560/aaf44b

83. Li L, Zhao X, Lu W, Tan S. Deep Learning for Variational Multimodality Tumor Segmentation in PET/CT. Neurocomputing (2020) 392:277-95. doi: 10.1016/j.neucom.2018.10.099 
84. Bi N, Wang J, Zhang T, Chen X, Xia W, Miao J, et al. Deep Learning Improved Clinical Target Volume Contouring Quality and Efficiency for Postoperative Radiation Therapy in Non-Small Cell Lung Cancer. Front Oncol (2019) 9:1192. doi: 10.3389/fonc.2019.01192

85. Aljabar P, Heckemann RA, Hammers A, Hajnal JV, Rueckert D. Multi-Atlas Based Segmentation of Brain Images: Atlas Selection and Its Effect on Accuracy. NeuroImage (2009) 46(3):726-38. doi: 10.1016/j.neuroimage.2009.02.018

86. Isgum I, Staring M, Rutten A, Prokop M, Viergever MA, Ginneken Bv. Multi-Atlas-Based Segmentation With Local Decision Fusion-Application to Cardiac and Aortic Segmentation in CT Scans. IEEE Trans Med Imaging (2009) 28(7):1000-10. doi: 10.1109/TMI.2008.2011480

87. Iglesias JE, Sabuncu MR. Multi-Atlas Segmentation of Biomedical Images: A Survey. Med Image Anal (2015) 24(1):205-19. doi: 10.1016/ j.media.2015.06.012

88. Qazi AA, Pekar V, Kim J, Xie J, Breen SL, Jaffray DA. Auto-Segmentation of Normal and Target Structures in Head and Neck CT Images: A Feature-Driven Model-Based Approach. Med Phys (2011) 38(11):6160-70. doi: 10.1118/1.3654160

89. Ecabert O, Peters J, Schramm H, Lorenz C, Berg Jv, Walker MJ, et al. Automatic Model-Based Segmentation of the Heart in CT Images. IEEE Trans Med Imaging (2008) 27(9):1189-201. doi: 10.1109/TMI.2008.918330

90. Sun S, Bauer C, Beichel R. Automated 3-D Segmentation of Lungs With Lung Cancer in CT Data Using a Novel Robust Active Shape Model Approach. IEEE Trans Med Imaging (2012) 31(2):449-60. doi: 10.1109/ TMI.2011.2171357

91. Lustberg T, van Soest J, Gooding M, Peressutti D, Aljabar P, van der Stoep J, et al. Clinical Evaluation of Atlas and Deep Learning Based Automatic Contouring for Lung Cancer. Radiother Oncol (2018) 126(2):312-7. doi: 10.1016/j.radonc.2017.11.012

92. Wang Z, Zou N, Shen D, Ji S. Non-Local U-Nets for Biomedical Image Segmentation. Proc AAAI Conf Artif Intell (2020) 34: (4):6315-22. doi: 10.1609/aaai.v34i04.6100

93. Vaswani A, Shazeer N, Parmar N, Uszkoreit J, Jones L, Gomez AN, et al. Attention Is All You Need, in: Proceedings of the 31st International Conference on Neural Information Processing Systems ( NIPS'17), Long Beach, California, USA: Curran Associates Inc, (2017). pp. 6000-10. doi: $10.5555 / 3295222.3295349$

94. Wang X, Girshick R, Gupta A, He K. (2018). Non-Local Neural Networks, in: 2018 IEEE/CVF Conference on Computer Vision and Pattern Recognition pp. 7794-803. doi: 10.1109/CVPR.2018.00813

95. Yuan H, Zou N, Zhang S, Peng H, Ji S. (2019). Learning Hierarchical and Shared Features for Improving 3D Neuron Reconstruction, in: 2019 IEEE International Conference on Data Mining (ICDM), 2019 Vol. 2019. pp. 80615. doi: 10.1109/ICDM.2019.00091

96. Salimans T, Goodfellow I, Zaremba W, Cheung V, Radford A, Chen X. Improved Techniques for Training GANs. In: Proceedings of the 30th
International Conference on Neural Information Processing Systems (NIPS'16). (2016). Curran Associates Inc.:Red Hook, NY, USA, p. 223442. https://dl.acm.org/doi/10.5555/3157096.3157346

97. Fechter T, Adebahr S, Baltas D, Ben Ayed I, Desrosiers C, Dolz J. Esophagus Segmentation in CT via 3D Fully Convolutional Neural Network and Random Walk. Med Phys (2017) 44(12):6341-52. doi: 10.1002/mp.12593

98. Yamashita H, Haga A, Hayakawa Y, Okuma K, Yoda K, Okano Y, et al. Patient Setup Error and Day-to-Day Esophageal Motion Error Analyzed by Cone-Beam Computed Tomography in Radiation Therapy. Acta Oncol (Stockholm Sweden) (2010) 49(4):485-90. doi: 10.3109/02841861003652574

99. Cohen RJ, Paskalev K, Litwin S, Price RAJr., Feigenberg SJ, Konski AA. Esophageal Motion During Radiotherapy: Quantification and Margin Implications. Dis Esophagus: Off J Int Soc Dis Esophagus (2010) 23(6):4739. doi: 10.1111/j.1442-2050.2009.01037.x

100. Palmer J, Yang J, Pan T, Court LE. Motion of the Esophagus Due to Cardiac Motion. PloS One (2014) 9(2):e89126. doi: 10.1371/journal.pone.0089126

101. Shin H, Roth HR, Gao M, Lu L, Xu Z, Nogues I, et al. Deep Convolutional Neural Networks for Computer-Aided Detection: CNN Architectures, Dataset Characteristics and Transfer Learning. IEEE Trans Med Imaging (2016) 35(5):1285-98. doi: 10.1109/TMI.2016.2528162

102. Men K, Chen X, Zhu J, Yang B, Zhang Y, Yi J, et al. Continual Improvement of Nasopharyngeal Carcinoma Segmentation With Less Labeling Effort. Phys Med: PM: Int J Devoted Appl Phys Med Biol: Off J Ital Assoc Biomed Phys (AIFB) (2020) 80:347-51. doi: 10.1016/j.ejmp.2020.11.005

103. Zhang S, Wang H, Tian S, Zhang X, Li J, Lei R, et al. A Slice Classification Model-Facilitated 3D Encoder-Decoder Network for Segmenting Organs at Risk in Head and Neck Cancer. J Radiat Res (2021) 62(1):94-103. doi: 10.1093/jrr/rraa094

104. Qin X, Zhang Z, Huang C, Dehghan M, Zaiane OR, Jagersand M. U2-Net: Going Deeper With Nested U-Structure for Salient Object Detection. Pattern Recognit (2020) 106:107404. doi: 10.1016/j.patcog.2020.107404

105. Cardenas CE, Yang J, Anderson BM, Court LE, Brock KB. Advances in AutoSegmentation. Semin Radiat Oncol (2019) 29(3):185-97. doi: 10.1016/ j.semradonc.2019.02.001

Conflict of Interest: The authors declare that the research was conducted in the absence of any commercial or financial relationships that could be construed as a potential conflict of interest.

Copyright (C) 2021 Liu, Li, Yang and Geng. This is an open-access article distributed under the terms of the Creative Commons Attribution License (CC BY). The use, distribution or reproduction in other forums is permitted, provided the original author(s) and the copyright owner(s) are credited and that the original publication in this journal is cited, in accordance with accepted academic practice. No use, distribution or reproduction is permitted which does not comply with these terms. 